\title{
A New Vortex Initialization Scheme Coupled with WRF-ARW
}

\author{
Jimmy Chi Hung Fung ${ }^{1,2}$ and Guangze Gao ${ }^{1}$ \\ ${ }^{1}$ Department of Mathematics, The Hong Kong University of Science and Technology, Clear Water Bay, Kowloon, Hong Kong \\ ${ }^{2}$ Division of Environment, The Hong Kong University of Science and Technology, Clear Water Bay, Kowloon, Hong Kong
}

Correspondence should be addressed to Jimmy Chi Hung Fung; majfung@ust.hk

Received 21 August 2016; Revised 29 October 2016; Accepted 20 November 2016; Published 3 January 2017

Academic Editor: Anthony R. Lupo

Copyright (C) 2017 J. C. H. Fung and G. Gao. This is an open access article distributed under the Creative Commons Attribution License, which permits unrestricted use, distribution, and reproduction in any medium, provided the original work is properly cited.

\begin{abstract}
The ability of numerical simulations to predict typhoons has been improved in recent decades. Although the track prediction is satisfactory, the intensity prediction is still far from adequate. Vortex initialization is an efficient method to improve the estimations of the initial conditions for typhoon forecasting. In this paper, a new vortex initialization scheme is developed and evaluated. The scheme requires only observational data of the radius of maximum wind and the max wind speed in addition to the global analysis data. This scheme can also satisfy the vortex boundary conditions, which means that the vortex is continuously merged into the background environment. The scheme has a low computational cost and has the flexibility to adjust the vortex structure. It was evaluated with 3 metrics: track, center sea-level pressure (CSLP), and maximum surface wind speed (MWSP). Simulations were conducted using the WRF-ARW numerical weather prediction model. Super and severe typhoon cases with insufficiently strong initial MWSP were simulated without and with the vortex initialization scheme. The simulation results were compared with the 6-hourly observational data from Hong Kong Observatory (HKO). The vortex initialization scheme improved the intensity (CSLP and MWSP) prediction results. The scheme was also compared with other initialization methods and schemes.
\end{abstract}

\section{Introduction}

To reduce the damage caused by tropical cyclones (TC), it is very important to predict their track and intensity correctly. In recent decades, track prediction has been steadily improved [1]. However, there has been little improvement in predicting the intensity of typhoons [1]. During 19892012, TC intensity errors from the best available models have decreased at $1 \%-2 \% \mathrm{yr}^{-1}$ at 24,48 , and $72 \mathrm{~h} \mathrm{[2].}$

As mentioned by Marks et al. [3], the primary reason for the slow progress in improvement of intensity prediction is that the intensity depends on the inner-core dynamics, whereas the track prediction depends more on the large-scale environments. The inner-core dynamics are mainly associated with vortex structure, intensity, and vortex size. It has been suggested that the accurate representation of the inner-core structure of tropical cyclones in the initial conditions is as important as the representation of the largescale environment [4]. To improve the representation of the initial vortex, a bogus vortex is often applied because the initial vortices provided by the large-scale analysis are often too weak or misplaced [5]. For example, for the super typhoon Genevieve (2014) at 12:00 (UTC) on 9 Aug 2014, the center sea-level pressure (CSLP) derived from FNL (NCEP final analyses) data is around $993 \mathrm{hPa}$, whereas the observational CSLP from $\mathrm{HKO}$ is $940 \mathrm{hPa}$.

Vortex initialization is an efficient method to provide better estimates of the initial conditions for numerical simulation of tropical cyclones. The advantages of the use of bogus vortices are the ability to customize the vortex structure and low computational cost, compared with dynamical initialization and variational data assimilation methods [6].

In Kwon and Cheong [7], an initialization method with an idealized 3D bogus vortex is developed for track and intensity predictions. In their scheme, a 3D axisymmetric bogus vortex is designed empirically in terms of analytic functions. The bogus vortex is used as a disturbance field and merged into the environmental field. The surface pressure profile of the bogus vortex is given by an exponent decay function. The temperature is derived from the $3 \mathrm{D}$ geopotential by using the 
hydrostatic balance equation. The relative humidity is given by an empirical function, which is not dynamically consistent with the other variables. In our scheme, this problem is overcome by solving the temperature and the humidity simultaneously (Equation (50)).

Reed and Jablonowski [8] used analytic functions to derive the initial conditions. The initial axisymmetric vortex is in hydrostatic and gradient wind balance. The first step is to provide the analytic background specific humidity profile:

$$
\begin{aligned}
& \bar{q}(z) \\
& \quad= \begin{cases}q_{0} \exp \left(-\frac{z}{z_{q 1}}\right) \exp \left[-\left(\frac{z}{z_{q 2}}\right)^{2}\right], & 0 \leq z \leq z_{t} ; \\
q_{t}, & z_{t}<z,\end{cases}
\end{aligned}
$$

where $z_{t}$ is set as $15 \mathrm{~km}$, which approximates the tropopause height; $q_{0}$ is the specific humidity at the surface, which is set as $21 \mathrm{~g} \mathrm{~kg}^{-1}$; and $q_{t}$ is the specific humidity in the upper atmosphere, which is set as $10^{-8} \mathrm{~g} \mathrm{~kg}^{-1} \cdot z_{q 1}=3000 \mathrm{~m}$ and $z_{q 2}=8000 \mathrm{~m}$ are two constants to control the specific humidity profile. The virtual temperature profile is given by

$$
\overline{T_{v}}(z)= \begin{cases}T_{v 0}-\Gamma z, & 0 \leq z \leq z_{t} \\ T_{v t}, & z_{t}<z\end{cases}
$$

where $T_{v 0}$ is the virtual potential temperature at the surface, and $\Gamma$ is the virtual temperature lapse rate, which is set as $0.007 \mathrm{~K} \mathrm{~m}^{-1} . T_{v t}=T_{v 0}-\Gamma z_{t}$ is the virtual temperature in the upper atmosphere. The background temperature profile can then be derived as

$$
\bar{T}(z)=\frac{\overline{T_{v}}(z)}{1+0.608 \bar{q}(z)} .
$$

The background pressure profile $\bar{p}(z)$ is computed using the hydrostatic equation and the ideal gas law. To obtain the perturbation profile of temperature, an empirical exponent decay function is used to modify the pressure perturbation. Then, by the hydrostatic equation and the ideal gas law, the temperature is derived. Finally, the tangential velocity $v_{T}(r, z)$ is derived by the gradient wind balance, which is given by

$$
\begin{aligned}
v_{T}(r, z)= & -\frac{f_{c} r}{2} \\
& +\left(\frac{f_{c}^{2} r^{2}}{4}+\frac{R_{d} T_{v}(r, z) r}{p(r, z)} \frac{\partial p(r, z)}{\partial r}\right)^{1 / 2},
\end{aligned}
$$

where $f_{c}=2 \Omega \sin \left(\varphi_{c}\right)$ is the Coriolis parameter at the constant latitude $\varphi_{c}$ and $\Omega=7.292 \times 10^{-5} \mathrm{~s}^{-1}$ is the rotational speed of Earth. $\varphi_{c}$ is set to be $10^{\circ} . r$ is distance to the TC center. $R_{d}=287.04 \mathrm{~J} \mathrm{~kg}^{-1} \mathrm{~K}^{-1}$ is the ideal gas constant for dry air. $T_{v}$ and $p$ are virtual temperature and pressure. The analytic formulation of this scheme enables it to be applied in various weather and climate models. However, the specific humidity is constructed as a horizontally homogeneous variable, which may not be realistic for the tropical cyclones. Also, the specific humidity is not adjustable, which means that the same specific humidity field must be used for all the tropical cyclones. Therefore, the scheme may be lacking flexibility to simulate different tropical cyclones.

In Rappin et al. [6], a highly configurable vortex initialization scheme is developed, which can be easily manipulated to generate different initial vortex structures. The vortex construction starts with parameterizations of the radial wind structure. The tangential wind $V(r)$ of the MR (modified Rankine) vortex is given by

$$
V(r)= \begin{cases}V_{\max } \frac{r}{\mathrm{RMW}}, & r \leq \mathrm{RMW} \\ V_{\max }\left(\frac{\mathrm{RMW}}{r}\right)^{\alpha}, & r>\mathrm{RMW}\end{cases}
$$

where $\alpha$ is the decaying factor, RMW is the radius of maximum wind, $V_{\max }$ is the tangential velocity at RMW, and $r$ is the distance from the TC center.

The vertical structure of tangential wind speed follows Stern and Nolan [9]:

$$
V(r, z)=V(r) \exp \left(-\frac{\left|z-z_{\max }\right|^{\gamma}}{\gamma L^{\gamma}}\right),
$$

where $\gamma$ is set as 2 and $L$ is set as $3175 \mathrm{~m}$ below $z_{\max }$ and $4762.5 \mathrm{~m}$ above $z_{\max }$ [9]. $z_{\max }$ is the height of the boundary layer top. Finally, the hydrostatic balance and gradient wind balance are used to determine temperature and pressure. One advantage of this scheme is that it makes use of (6), which considers the vertical structure of tangential wind speed. By using this equation, the $3 \mathrm{D}$ wind field can be derived from a 2D wind structure (e.g., the MR profile) directly, which makes the scheme highly configurable. The equations for constructing horizontal and vertical profiles introduced in Rappin et al. [6] may also be helpful in designing new schemes. In our new scheme, we further improve the wind construction by making the decaying factor and RMW as functions of $z$.

In this paper, a vortex initialization scheme is developed for the weather research and forecasting (WRF) model with the advanced research WRF (ARW) core. Further, in our scheme, the variables satisfy geophysical formulas, which ensures that the variables are dynamically consistent. The analytic formulations of this scheme enable it to be conducted efficiently. The problems in other schemes mentioned above can also be solved by our scheme. Notice that these problems are mainly associated with two aspects: (1) the variables should be dynamically consistent with each other; (2) the TCs constructed by a scheme should be adjustable and realistic.

The vortex initialization problem can be viewed as follows: given the data sets of $(u, v, p, T, q$, etc.) from global analysis data (e.g., FNL and GFS (global forecast system) data), how can we correct them to new data $\left(u^{*}, v^{*}, p^{*}, T^{*}, q^{*}\right.$, etc. $)$ ? Here, $u, v, p, T, q$ represent EW-wind, NS-wind, pressure, temperature, and specific humidity, respectively; “*” is used to represent corrected data throughout this paper. In most situations, we need to provide some extra data besides the global analysis data to build the new vortex. For example, we may need the max wind speed and RMW. Although providing 
more data may help us to achieve better initial fields, it is difficult to obtain observational data on the ocean. In our scheme, we aim to construct a $3 \mathrm{D}$ vortex that can represent basic features of typhoon's structure. The extra inputs are vortex location, max wind speed, and the radius of the max wind.

The rest of the paper is organized as follows: in Section 2, the vortex initialization scheme is derived; in Section 3, numerical simulations for super and severe typhoons in the northwestern Pacific are conducted to evaluate the performance of the new scheme; the simulation results are discussed in Section 4; and in Section 5, conclusions are given.

\section{The New Vortex Initialization Scheme}

2.1. 2D Wind Correction. The goal in this paper is to construct a new 3D vortex. Construction of one vertical layer is first discussed, which is started from wind construction. The wind construction can be disaggregated as size correction, intensity correction, and computation of the intensity correction factor.

2.1.1. Size and Intensity Correction. The first step is "size correction" [13]. If the max wind speed and the RMW are computed by the FNL data directly, the max wind speed is generally smaller than the observational data, and the RMW is generally too large. The typhoon size can be corrected by compressing the model grids. $r$ and $r^{*}$ are the distances from a grid point to the typhoon center, before and after size correction. The size correction is conducted by

$$
\begin{array}{ll}
u_{1}^{*}\left(r^{*}, \theta, z\right)=u(r, \theta, z), & \left(\text { if } r^{*} \leq R_{m}^{*}\right) ; \\
v_{1}^{*}\left(r^{*}, \theta, z\right)=v(r, \theta, z), & \left(\text { if } r^{*} \leq R_{m}^{*}\right),
\end{array}
$$

where $u_{1}^{*}$ and $v_{1}^{*}$ are the wind components after size correction. $\theta$ is the azimuthal angle and $z$ is the height from the ground. This means that the wind speed with distance $r$ is moved to the position with distance $r^{*}$. In particular, the wind speeds with distance $r_{m}$ (RMW) and $R_{m}$ (vortex size) are moved to new positions with distance $r_{m}^{*}$ and $R_{m}^{*}$, respectively. The relationship between $r$ and $r^{*}$ is given by the assumption of linear compression as follows:

$$
\begin{aligned}
\frac{d r^{*}}{d r} & =a+b r, \\
r^{*}\left(r_{m}\right) & =r_{m}^{*}, \\
r^{*}\left(R_{m}\right) & =R_{m}^{*}, \\
r^{*}(0) & =0 .
\end{aligned}
$$

It can deduced that

$$
\begin{aligned}
& a=\frac{r_{m}^{*} R_{m}^{2}-r_{m}^{2} R_{m}^{*}}{R_{m} r_{m}\left(R_{m}-r_{m}\right)}, \\
& b=2 \frac{R_{m}^{*} r_{m}-R_{m} r_{m}^{*}}{R_{m} r_{m}\left(R_{m}-r_{m}\right)} .
\end{aligned}
$$

In practice, the vortex boundary is defined as the contour line of a sea-level pressure value (e.g., $1013 \mathrm{hPa}$ ). After size correction, the new vortex is called $u_{1}^{*}$-vortex.

The second step is "intensity correction" [13]. As mentioned by Lord [14], the vortex initialization is usually carried out by first implanting a synthetic vortex into the large-scale analysis. Similarly, the intensity correction in this paper is conducted by superposing an axisymmetric vortex onto $u_{1}^{*}$-vortex. The axisymmetric vortex is called $u_{2}$ vortex. When adding $u_{2}$-vortex to $u_{1}^{*}$-vortex, the intensity is adjusted through the intensity correction factor. In the next subsection, the issues related to the computation of the intensity correction factor are addressed.

In the axisymmetric $u_{2}$-vortex, the tangential velocity is $V(r, z)$, and the radial velocity is zero. $V(r, z)$ is defined as follows: denote $V_{m}(z)$ as the max tangential wind speed at height $z$ in the axisymmetric vortex, which is the same as the max wind magnitude in the original vortex and $u_{1}^{*}$-vortex. $V(r, z)$ is the tangential velocity on the circle of radius $r$ (the center of the circle is the TC center). $V(r, z)$ is defined in the form of an MR vortex:

$$
\begin{aligned}
& V(r, z) \\
& \quad= \begin{cases}V_{m}(z) \frac{r}{r_{m}^{*}(z)}, & 0 \leq r \leq r_{m}^{*}(z) ; \\
V_{m}(z)\left(\frac{r_{m}^{*}(z)}{r}\right)^{\alpha(z)}, & r_{m}^{*}(z)<r \leq R_{m}^{*}(z) ; \\
0, & r>R_{m}^{*}(z),\end{cases}
\end{aligned}
$$

where $\alpha(z)$ is the profile decay rate, which is determined later. For variable $a$, define

$$
\langle a\rangle=\frac{1}{2 \pi} \int_{0}^{2 \pi} a(r, \theta, z) d \theta .
$$

The physical meaning of $\langle a\rangle$ is the azimuthal mean of variable $a$. By using this symbol, define

$$
\begin{aligned}
V_{m}(z) & =V\left(r_{m}^{*}, z\right) \\
& =\sqrt{\left\langle u^{2}\left(r_{m}(z), \theta, z\right)+v^{2}\left(r_{m}(z), \theta, z\right)\right\rangle}, \\
V\left(R_{m}^{*}, z\right) & =\sqrt{\left\langle u^{2}\left(R_{m}(z), \theta, z\right)+v^{2}\left(R_{m}(z), \theta, z\right)\right\rangle} .
\end{aligned}
$$

Then, the decay rate can be deduced:

$$
\begin{aligned}
V\left(r_{m}^{*}, z\right) & =V_{m}(z), \\
V\left(R_{m}^{*}, z\right) & =V_{m}(z)\left(\frac{r_{m}^{*}}{R_{m}^{*}}\right)^{\alpha(z)}, \\
\alpha(z) & =\log _{R_{m}^{*}(z) / r_{m}^{*}(z)} \frac{V_{m}(z)}{V\left(R_{m}^{*}, z\right)} .
\end{aligned}
$$

Azimuthal means are used to compute $\alpha(z)$ because this is more representative than the use of single-point estimations. Then, $u_{2}(r, \theta, z)=-V(r, z) \sin (\theta), v_{2}(r, \theta, z)=V(r, z) \cos (\theta)$. Here, $\theta$ is the angle between $x$-axis and the radial directional 
vector; $u_{2}$ and $v_{2}$ are the EW- and NS-wind components of $u_{2}$-vortex. In the final step, the wind is corrected by

$$
\begin{aligned}
& u^{*}(r, \theta, z)=u_{1}^{*}(r, \theta, z)+\beta(r, z) u_{2}(r, \theta, z) ; \\
& v^{*}(r, \theta, z)=v_{1}^{*}(r, \theta, z)+\beta(r, z) v_{2}(r, \theta, z),
\end{aligned}
$$

where $u^{*}$ and $v^{*}$ are the functions of corrected wind and $\beta$ is the intensity correction factor.

2.1.2. Computation of the Intensity Correction Factor. In this subsection, the function of $\beta$ (the intensity correction factor) is determined. To simplify notations, let $\beta_{1}(z)=$ $\beta\left(r_{m}^{*}(z), z\right), \beta_{2}(z)=\beta\left(R_{m}^{*}(z), z\right)$, and $\lambda(z)=\left(\beta_{2}(z)-\right.$ $\left.\beta_{1}(z)\right) /\left(R_{m}^{*}(z)-r_{m}^{*}(z)\right)$. The following equation shows that the max wind speed at RMW is intensified to be the same as the observational max wind:

$$
\begin{gathered}
V_{\mathrm{obs}}(z)^{2}=\left(u^{*}\left(r_{m}^{*}(z), \theta_{m}^{*}(z), z\right)\right)^{2} \\
+\left(v^{*}\left(r_{m}^{*}(z), \theta_{m}^{*}(z), z\right)\right)^{2}, \\
V_{\mathrm{obs}}(z)^{2}=\left(u_{1}^{*}\left(r_{m}^{*}(z), \theta_{m}^{*}(z), z\right)\right. \\
\left.+\beta_{1}(z) u_{2}\left(r_{m}^{*}(z), \theta_{m}^{*}(z), z\right)\right)^{2} \\
+\left(v_{1}^{*}\left(r_{m}^{*}(z), \theta_{m}^{*}(z), z\right)\right. \\
\left.+\beta_{1}(z) v_{2}\left(r_{m}^{*}(z), \theta_{m}^{*}(z), z\right)\right)^{2},
\end{gathered}
$$

where $\theta_{m}^{*}(z)$ is the corresponding angle at RMW with height $z$. The vertical profile of $V_{\text {obs }}(z)$ is specified later. Then the following equation can be deduced:

$$
\beta_{1}(z)=\left.\frac{-u_{1}^{*} u_{2}-v_{1}^{*} v_{2}+\sqrt{V_{\mathrm{obs}}^{2}\left(u_{2}^{2}+v_{2}^{2}\right)-\left(u_{1}^{*} v_{2}-v_{1}^{*} u_{2}\right)^{2}}}{u_{2}^{2}+v_{2}^{2}}\right|_{\left(r_{m}^{*}(z), \theta_{m}^{*}(z), z\right)}
$$

The next step is to deduce $\beta_{2}(z)$, which is the intensity correction factor at $R_{m}^{*}(z)$. The reasoning is based on the fact that the typhoon vortex should be merged with the environment continuously. In other words, the vortex boundary values should be close to those of the large-scale background. Mathematically, on the vortex boundary of a typhoon, all of the variables should be continuous. Now, consider a radial direction with the corresponding angle $\theta_{m}$. By the boundary continuity,

$$
\lim _{r \rightarrow R_{m}^{*-}} u^{*}\left(r, \theta_{m}, z\right)=\lim _{r \rightarrow R_{m}^{*+}} u^{*}\left(r, \theta_{m}, z\right)
$$

Note that $\lim _{r \rightarrow R_{m}^{*-}} u^{*}\left(r, \theta_{m}, z\right)=u^{*}\left(R_{m}^{*}, \theta_{m}, z\right)$, which is the EW-wind component at the vortex boundary after correction. On the other hand, $\lim _{r \rightarrow R_{m}^{*+}} u^{*}\left(r, \theta_{m}, z\right)=$ $\lim _{r \rightarrow R_{m}^{*+}}\left(u_{1}^{*}\left(r, \theta_{m}, z\right)+\beta_{2} u_{2}\left(r, \theta_{m}, z\right)\right)=$ $\lim _{r \rightarrow R_{m}^{*+}} u_{1}^{*}\left(r, \theta_{m}, z\right)=\lim _{r \rightarrow R_{m}^{*+}} u\left(r, \theta_{m}, z\right)=u\left(R_{m}^{*}, \theta_{m}, z\right)$. Recall that $u\left(R_{m}^{*}, \theta_{m}, z\right)$ is the EW-wind component at the vortex boundary before correction. The same arguments can be applied to $v$. Therefore, at the boundary of the vortex, the wind magnitude after the change should be equal to the wind magnitude before the change. Choose point $B_{0}$ on the vortex boundary before size correction. After size correction, $B_{0}$ becomes $B$. Consider the wind speed at $B$ :

$$
\begin{aligned}
u^{* 2} & \left(R_{m}^{*}, \theta_{B}, z\right)+v^{* 2}\left(R_{m}^{*}, \theta_{B}, z\right) \\
& =u^{2}\left(R_{m}^{*}, \theta_{B}, z\right)+v^{2}\left(R_{m}^{*}, \theta_{B}, z\right) .
\end{aligned}
$$

This can be expanded as

$$
\begin{aligned}
\left(u_{1}^{*}\right. & \left.\left(R_{m}^{*}, \theta_{B}, z\right)+\beta_{2}(z) u_{2}\left(R_{m}^{*}, \theta_{B}, z\right)\right)^{2} \\
& \quad+\left(v_{1}^{*}\left(R_{m}^{*}, \theta_{B}, z\right)+\beta_{2}(z) v_{2}\left(R_{m}^{*}, \theta_{B}, z\right)\right)^{2} \\
= & u^{2}\left(R_{m}^{*}, \theta_{B}, z\right)+v^{2}\left(R_{m}^{*}, \theta_{B}, z\right) .
\end{aligned}
$$

Therefore,

$$
\begin{aligned}
(u & \left.\left(R_{m}, \theta_{B}, z\right)+\beta_{2}(z) u_{2}\left(R_{m}^{*}, \theta_{B}, z\right)\right)^{2} \\
& +\left(v\left(R_{m}, \theta_{B}, z\right)+\beta_{2}(z) v_{2}\left(R_{m}^{*}, \theta_{B}, z\right)\right)^{2} \\
= & u^{2}\left(R_{m}^{*}, \theta_{B}, z\right)+v^{2}\left(R_{m}^{*}, \theta_{B}, z\right) .
\end{aligned}
$$

Because $u$ and $u_{2}$ are known, the above equation for $\beta_{2}$ can be solved numerically. Here, an analytic approximation for $\beta_{2}$ is introduced, which can make the computations more efficient.

$$
\begin{aligned}
u_{2} & \left(R_{m}^{*}, \theta_{B}, z\right)=-V\left(R_{m}^{*}, z\right) \sin \left(\theta_{B}\right) \\
& =-\sqrt{u^{2}\left(R_{m}, z\right)+v^{2}\left(R_{m}, z\right)} \sin \left(\theta_{B}\right) \\
& \approx-\sqrt{u^{2}\left(R_{m}, \theta_{B}, z\right)+v^{2}\left(R_{m}, \theta_{B}, z\right)} \sin \left(\theta_{B}\right) \\
& =-\sqrt{V_{B}^{2}+U_{B}^{2}} \sin \left(\theta_{B}\right) \approx-V_{B} \sin \left(\theta_{B}\right) \\
& \approx-V_{B} \sin \left(\theta_{B}\right)+U_{B} \cos \left(\theta_{B}\right) \\
& =u\left(R_{m}, \theta_{B}, z\right),
\end{aligned}
$$

where $V_{B}$ is the tangential wind at $B$, which is counterclockwise. $U_{B}$ is the radial wind at $B$, which is outward. In the 
above approximations, two facts are applied: (1) the wind's magnitude changes a little as $\theta$ changes; and (2) the radial wind is small, compared with the tangential wind. Similarly, $v_{2}\left(R_{m}^{*}, \theta_{B}, z\right)=v\left(R_{m}, \theta_{B}, z\right)$. Therefore,

$$
\beta_{2}(z)=\sqrt{\frac{u^{2}\left(R_{m}^{*}(z), \theta_{B}, z\right)+v^{2}\left(R_{m}^{*}(z), \theta_{B}, z\right)}{u^{2}\left(R_{m}(z), \theta_{B}, z\right)+v^{2}\left(R_{m}(z), \theta_{B}, z\right)}}-1 .
$$

In practice, azimuthal means are used to estimate $\beta_{2}$, and the approximation formula is as follows:

$$
\begin{aligned}
\beta_{2}(z)= & \sqrt{\frac{\left\langle u^{2}\left(R_{m}^{*}(z), \theta, z\right)+v^{2}\left(R_{m}^{*}(z), \theta, z\right)\right\rangle}{\left\langle u^{2}\left(R_{m}(z), \theta, z\right)+v^{2}\left(R_{m}(z), \theta, z\right)\right\rangle}} \\
& -1 .
\end{aligned}
$$

The above estimation is deduced under an assumption of wind continuity. Further conclusions, considering the continuity of other variables, are demonstrated in later sections. Finally, the formula of $\beta(r, z)$ is given. Because of sparse data, let $\beta$ be a piecewise linear function:

$$
\begin{aligned}
& \beta(r, z) \\
& = \begin{cases}\beta_{1}(z), & 0 \leq r \leq r_{m}^{*}(z) ; \\
\beta_{1}(z)+\lambda(z)\left(r-r_{m}^{*}(z)\right), & r_{m}^{*}(z)<r \leq R_{m}^{*}(z) ; \\
0, & r>R_{m}^{*}(z) .\end{cases}
\end{aligned}
$$

By following all the steps above, the $2 \mathrm{D}$ wind fields $u^{*}$ and $v^{*}$ can be constructed if the observational max wind speed is provided. In later sections, the vertical profile of $V_{\mathrm{obs}}$ is demonstrated and the 3D wind field is deduced.

2.2. $3 D$ Wind Correction. The inputs to correct the vertical layer at height $z$ are $r_{m}^{*}(z), R_{m}^{*}(z)$, and $V_{\text {obs }}(z)$. Once these three profiles are provided, the $3 \mathrm{D}$ wind fields can be corrected. In practice, $R_{m}^{*}(z)=R_{m}(z)$, which can be deduced from the continuous vortex boundary conditions in later sections. In this section, the focus is on solving $r_{m}^{*}(z)$ and $V_{\text {obs }}(z)$. In Rappin et al. [6] and Stern and Nolan [9], the vertical wind structure is constructed as

$$
V(r, z)=V(r) \exp \left(-\frac{\left|z-z_{\max }\right|^{\gamma}}{\gamma L^{\gamma}}\right),
$$

where $V(r)$ is the tangential wind speed at $r$ and $z_{\max }, V(r, z)$ is the tangential wind speed at $r$ and $z, z_{\max }$ is the height of the maximum wind, $L$ is the vertical depth scale, and $\gamma$ is the parameter that controls the decay rate. This profile is an important result in Stern and Nolan [15]. Change (25) to

$$
V_{\text {obs }}(z)=V_{\text {obs }}\left(z_{\text {max }}\right) \exp \left(-\frac{\left|z-z_{\max }\right|^{\gamma}}{\gamma L^{\gamma}}\right) .
$$

The reasons for this are as follows: (1) the radial wind is smaller than the tangential wind, so the magnitude of the horizontal wind is dominated by the tangential wind; (2) in a typhoon, the radial wind above $3 \mathrm{~km}$ is very small (typically less than $\left.1 \mathrm{~m} \mathrm{~s}^{-1}\right)$. Notice that new parameters introduced here are $V_{\text {obs }}\left(z_{\text {max }}\right), \gamma$, and $L$ (a height related to the decay rate). $\gamma$ is set as 2 in Stern and Nolan [9]. By estimating the observational data, Stern and Nolan [9] set parameter $L$ as $3175 \mathrm{~m}$ below $z_{\max }$ and $4762.5 \mathrm{~m}$ above $z_{\max }$.

Stern and Nolan [15] also confirmed that the absolute angular momentum at the RMW for different heights was almost a constant. Now use $V_{\text {obs }}(z)$ to represent the tangential wind in reverse:

$$
\begin{aligned}
M(z) & =V_{\text {obs }}(z) r_{m}^{*}(z)+\frac{1}{2} f_{0} r_{m}^{*}(z)^{2}, \\
M\left(z_{\text {max }}\right) & =V_{\text {obs }}\left(z_{\text {max }}\right) r_{m}^{*}\left(z_{\text {max }}\right)+\frac{1}{2} f_{0} r_{m}^{*}\left(z_{\text {max }}\right)^{2}, \\
M(z) & =M\left(z_{\text {max }}\right) .
\end{aligned}
$$

Therefore,

$$
r_{m}^{*}(z)=\frac{1}{f_{0}}\left[-V_{\mathrm{obs}}(z)+\sqrt{V_{\mathrm{obs}}^{2}(z)+2 M\left(z_{\mathrm{max}}\right) f_{0}}\right] .
$$

The 3D wind can be corrected from (26) and (28).

2.3. 3D Pressure Correction. The CSLP is an important metric, which is always used to evaluate the quality of tropical cyclone simulations. In this section, the gradient wind balance is used to deduce the pressure from the wind. This method is especially efficient when the wind fields are constructed analytically. By the gradient wind balance of the governing equation for the axisymmetric component, the following approximation can be deduced:

$$
\frac{1}{\rho} \frac{\partial p}{\partial r}=\frac{V^{2}}{r}+f_{0} V
$$

where $V$ is constructed with the same max wind speed and vortex boundary value as the original vortex. Therefore, its profile is used as the axisymmetric part of $u_{1}^{*}$-vortex. Because $u^{*}=u_{1}^{*}+\beta u_{2},(1+\beta) V$ is used as the axisymmetric part of the corrected vortex. $V$ corresponds to $u_{1}^{*}$, and $\beta V$ corresponds to $\beta u_{2}$. These two profiles have the same directions on the same grids, so they can be superimposed. The sea-level pressure correction step is based on the gradient wind stream function $\psi(r, z)$, which satisfies

$$
\frac{\partial \psi}{\partial r}=\frac{V^{2}}{r f_{0}}+V
$$

where $f_{0}$ is the Coriolis parameter at the TC center. Then,

$$
\psi=\int_{\infty}^{r}\left(\frac{V^{2}}{r f_{0}}+V\right) d r=\int_{R_{m}^{*}}^{r}\left(\frac{V^{2}}{r f_{0}}+V\right) d r
$$

The new gradient wind stream function is

$$
\psi^{*}=\int_{\infty}^{r}\left[\frac{((1+\beta) V)^{2}}{r f_{0}}+(1+\beta) V\right] d r
$$


Let $P_{e}$ be the environmental sea-level pressure. The profiles of the environmental pressure are given by the following equations:

$$
\begin{aligned}
\Theta & =\Theta^{\prime}+300, \\
T_{k} & =\Theta\left(\frac{p_{0}}{p}\right)^{-2 / 7}, \\
T_{v} & =T_{k}(1+\delta q), \\
p_{e}(z) & =\left.P_{e} \exp \left(-\frac{g z}{R_{d} T_{v}}\right)\right|_{R_{m}^{*}(z)},
\end{aligned}
$$

where $\Theta^{\prime}$ is the perturbed virtual temperature, which can be obtained from the outputs of WRF-ARW. Let $\Delta p(r, \theta, z)=$ $p(r, \theta, z)-p_{e}(z)$ and $\Delta p^{*}(r, \theta, z)=p^{*}(r, \theta, z)-p_{e}(z)$ be the pressure perturbations before and after correction, respectively. Assuming that $\rho$ and $f_{0}$ are constants, then,

$$
\begin{aligned}
\int_{\infty}^{r} \frac{1}{\rho} \frac{\partial p}{\partial r} d r & =\int_{\infty}^{r} f_{0} \frac{\partial \psi}{\partial r} d r \\
\frac{1}{\rho} \Delta p & =f_{0} \psi \\
\frac{1}{\rho} \Delta p^{*} & =f_{0} \psi^{*} .
\end{aligned}
$$

This shows that the pressure perturbation is proportional to the gradient wind stream function. The new pressure perturbation is

$$
\Delta p^{*}=\Delta p \frac{\psi^{*}}{\psi}
$$

The explicit formulations are

$$
\begin{aligned}
& \psi(r, z)=\frac{V_{m}(z)^{2} r^{2}}{2 f_{0}\left(r_{m}^{*}(z)\right)^{2}}+\frac{V_{m}(z) r^{2}}{2 r_{m}^{*}(z)}-\frac{V_{m}(z)^{2}}{2 f_{0}}-\frac{1}{2} \\
& \cdot V_{m}(z)+\psi\left(r_{m}^{*}(z), z\right), \quad r<r_{m}^{*}(z) ; \\
& \psi(r, z)=-\frac{1}{2 \alpha(z)} \frac{V_{m}(z)^{2}\left(r_{m}^{*}(z)\right)^{2 \alpha(z)}}{f_{0} r^{2 \alpha(z)}} \\
& +\frac{V_{m}(z)\left(r_{m}^{*}(z)\right)^{\alpha(z)}}{(1-\alpha(z)) r^{\alpha(z)-1}}+\frac{1}{2 \alpha(z)} \\
& \cdot \frac{V_{m}(z)^{2}\left(r_{m}^{*}(z)\right)^{2 \alpha(z)}}{f_{0}\left(R_{m}^{*}(z)\right)^{2 \alpha(z)}} \\
& -\frac{V_{m}(z)\left(r_{m}^{*}(z)\right)^{\alpha(z)}}{(1-\alpha(z))\left(R_{m}^{*}(z)\right)^{\alpha(z)-1}}, \\
& \quad r_{m}^{*}(z) \leq r \leq R_{m}^{*}(z) ;
\end{aligned}
$$

$$
\begin{aligned}
& \psi(r, z)=0, \quad r>R_{m}^{*}(z), \\
& \psi^{*}(r, z)=\frac{\left(1+\beta_{1}(z)\right)^{2} V_{m}(z)^{2} r^{2}}{2 f_{0}\left(r_{m}^{*}(z)\right)^{2}} \\
& +\frac{\left(1+\beta_{1}(z)\right) V_{m}(z) r^{2}}{2 r_{m}^{*}(z)}-\frac{\left(1+\beta_{1}(z)\right)^{2} V_{m}(z)^{2}}{2 f_{0}} \\
& -\frac{1}{2}\left(1+\beta_{1}(z)\right) V_{m}(z) r_{m}^{*}(z)+\psi^{*}\left(r_{m}^{*}(z), z\right) \text {, } \\
& r<r_{m}^{*}(z) ; \\
& \psi^{*}(r, z)=\left\{\frac { V _ { m } ( z ) ^ { 2 } ( r _ { m } ^ { * } ( z ) ) ^ { 2 \alpha ( z ) } } { f _ { 0 } r ^ { 2 \alpha ( z ) } } \left[\frac{\lambda(z)^{2} r^{2}}{2-2 \alpha(z)}\right.\right. \\
& +\frac{2 \lambda(z) r\left(1+\beta_{1}(z)-\lambda(z) r_{m}^{*}(z)\right)}{1-2 \alpha(z)} \\
& \left.-\frac{\left(1+\beta_{1}(z)-\lambda(z) r_{m}^{*}(z)\right)^{2}}{2 \alpha(z)}\right]+V_{m}(z) \\
& \cdot\left(r_{m}^{*}(z)\right)^{\alpha(z)} r^{1-\alpha(z)}\left(\frac{\lambda(z) r}{2-\alpha(z)}\right. \\
& \left.\left.+\frac{1+\beta_{1}(z)-\lambda(z) r_{m}^{*}(z)}{1-\alpha(z)}\right)\right\}\left.\right|_{R_{m}^{*}(z)} ^{r}, \\
& r_{m}^{*}(z) \leq r \leq R_{m}^{*}(z) ; \\
& \psi^{*}(r, z)=0, \quad r>R_{m}^{*}(z) .
\end{aligned}
$$

Finally, $p^{*}(r, \theta, z)=\left[\psi^{*}(r, z) / \psi(r, z)\right]\left[p(r, \theta, z)-p_{e}(z)\right]+$ $p_{e}(z)$ is the desired pressure profile.

2.4. 3D Temperature and Humidity Correction. In this subsection, a method to construct a $3 \mathrm{D}$ virtual temperature field $\left(T_{v}^{*}\right)$ is first introduced [13]. The key technique is to divide the total virtual temperature field into an environmental field and a vortex field. The environmental field provides the base state, and the vortex field provides the perturbations. From the hydrostatic equation and the ideal gas law, the following equation is obtained:

$$
\frac{\partial p}{\partial z}=-\frac{p}{R_{d} T_{v}} g
$$

where $R_{d}=287.04 \mathrm{~J} \mathrm{~kg}^{-1} \mathrm{~K}^{-1}$. Let $H$ be the height of the top of the typhoon vortex. $z_{0}$ is a fixed height. Integrate the above equation along the segment $(x, y) \times\left[z_{0}, H\right]$. In the environment,

$$
\ln \frac{p_{0}}{p_{t}}=\frac{g}{R_{d}} \int_{z_{0}}^{H} \frac{d z}{\overline{T_{v}}(z)},
$$

where $p_{0}, p_{t}$ are the pressures at $z_{0}$ and the top of the computational domain, respectively. $\overline{T_{v}}$ is the virtual temperature of the environment. In the total field,

$$
\ln \frac{p_{0}+\Delta p_{0}}{p_{t}}=\frac{g}{R_{d}} \int_{z_{0}}^{H} \frac{d z}{\overline{T_{v}}(z)+\Delta T_{v}(z)},
$$

where " $\Delta$ " signifies perturbation in the vortex, and the subscript " 0 " signifies the value at $z_{0} . \Delta T_{v}$ is the enhancement 
made to the original environment by the vortex part. Then the equation derived in the total field is modified as

$$
\begin{aligned}
\ln \frac{p_{0}}{p_{t}} \cdot\left(1+\frac{\Delta p_{0}}{p_{0}}\right) & =\frac{g}{R_{d}} \int_{z_{0}}^{H} \frac{d z}{\overline{T_{v}}(z)+\Delta T_{v}(z)} \\
& \approx \frac{g}{R_{d}} \int_{z_{0}}^{H} \frac{1}{\overline{T_{v}}}\left(1-\frac{\Delta T_{v}}{\overline{T_{v}}}\right) d z .
\end{aligned}
$$

Use this equation and the equation for the environment, and an approximation can be obtained for the perturbation of the virtual temperature:

$$
\ln \left(1+\frac{\Delta p_{0}}{p_{0}}\right) \approx-\frac{g}{R_{d}} \int_{z_{0}}^{H} \frac{\Delta T_{v}}{{\overline{T_{v}}}^{2}} d z .
$$

Because $\ln \left[1+\left(\Delta p_{0}\right) / p_{0}\right] \approx\left(\Delta p_{0}\right) / p_{0}$,

$$
\frac{\Delta p_{0}}{p_{0}}=-\frac{g}{R_{d}} \int_{z_{0}}^{H} \frac{\Delta T_{v}}{\bar{T}_{v}^{2}} d z .
$$

After correction,

$$
\frac{\Delta p_{0}^{*}}{p_{0}}=-\frac{g}{R_{d}} \int_{z_{0}}^{H} \frac{\Delta T_{v}^{*}}{\bar{T}_{v}^{2}} d z .
$$

Define $\Gamma(r, z)=\psi^{*}(r, z) / \psi(r, z)$. Recall that $\Delta p_{0}^{*}=$ $\Gamma\left(r, z_{0}\right) \Delta p_{0}$. Therefore,

$$
\begin{gathered}
\frac{\Gamma\left(r, z_{0}\right) \Delta p_{0}}{p_{0}}=-\frac{g}{R_{d}} \int_{z_{0}}^{H} \frac{\Gamma\left(r, z_{0}\right) \Delta T_{v}}{\bar{T}_{v}^{2}} d z, \\
\int_{z_{0}}^{H} \frac{\Delta T_{v}^{*}}{{\overline{T_{v}}}^{2}} d z=\int_{z_{0}}^{H} \frac{\Gamma\left(r, z_{0}\right) \Delta T_{v}}{\bar{T}_{v}^{2}} d z .
\end{gathered}
$$

For a fixed $z_{0}, \Delta T_{v}^{*}(z)=\Gamma\left(r, z_{0}\right) \Delta T_{v}(z)$ for any $z \in\left[z_{0}, H\right]$ is one simple solution. In particular, $\Delta T_{v}^{*}(z)=\Gamma(r, z) \Delta T_{v}(z)$. Now, $T_{v}^{*}$ is known. Between virtual temperature and temperature, they are connected by

$$
T^{*}(r, \theta, z)=\frac{T_{v}^{*}(r, \theta, z)}{1+\delta q^{*}(r, \theta, z)},
$$

where $\delta=0.608$. During the corrections, $q^{*} / e_{s}^{*}=q / e_{s}$ is assumed, where $e$ and $e_{s}(T)$ are vapor pressure and saturation vapor pressure. By definition, $q=(0.622 e) /(p-0.378 e)$, which is the specific humidity. Then, $q^{*}=\left(e_{s}^{*} / e_{s}\right) q$. From the definition of saturation vapor pressure,

$$
\begin{aligned}
e_{s}(T) & =6.112 \exp \left(17.67 \frac{T-273.16}{T-29.66}\right), \\
e_{s}^{*}\left(T^{*}\right) & =6.112 \exp \left(17.67 \frac{T^{*}-273.16}{T^{*}-29.66}\right) .
\end{aligned}
$$

It is obtained that

$$
\frac{e_{s}^{*}\left(T^{*}\right)}{e_{s}(T)}=\exp \left[\frac{17.67 \cdot 243.5\left(T^{*}-T\right)}{\left(T^{*}-29.66\right)(T-29.66)}\right] .
$$

The corrected humidity is

$$
q^{*}=\exp \left[\frac{17.67 \cdot 243.5\left(T^{*}-T\right)}{\left(T^{*}-29.66\right)(T-29.66)}\right] q \text {. }
$$

Equations (46) and (49) are two equations about $q^{*}$ and $T^{*}$. Combine them and notice that $T_{v}^{*}$ and $q$ are known:

$$
\frac{1}{\delta}\left(\frac{T_{v}^{*}}{T^{*}}-1\right)=q \exp \left[\frac{17.67 \cdot 243.5\left(T^{*}-T\right)}{\left(T^{*}-29.66\right)(T-29.66)}\right]
$$

The above pointwise equation is solved on each grid point within the vortex. Now, the $3 \mathrm{D}$ temperature and humidity fields are corrected.

2.5. Vortex Boundary Conditions. Pressure, temperature, and specific humidity should also be continuously merged into the environment. In other words, at the vortex boundary, the following equations should be valid:

$$
\begin{aligned}
& \lim _{r \rightarrow R_{m}^{*-}} p^{*}(r, \theta, z)=p\left(R_{m}^{*}, \theta, z\right), \\
& \lim _{r \rightarrow R_{m}^{*-}} T^{*}(r, \theta, z)=T\left(R_{m}^{*}, \theta, z\right), \\
& \lim _{r \rightarrow R_{m}^{*-}} q^{*}(r, \theta, z)=q\left(R_{m}^{*}, \theta, z\right) .
\end{aligned}
$$

The continuity of virtual temperature can also be deduced from the continuity of temperature and humidity:

$$
\begin{aligned}
T_{v}^{*}=T^{*}\left(1+\delta q^{*}\right) \longrightarrow T(1+\delta q)=T_{v}, & \\
& \left(r \longrightarrow R_{m}^{*-}\right) .
\end{aligned}
$$

Equation (51) is equivalent to

$$
\lim _{r \rightarrow R_{m}^{*-}} \frac{\psi^{*}(r, z)}{\psi(r, z)}=1
$$

Because $\lim _{r \rightarrow R_{m}^{*-}} \psi^{*}(r, z)=0, \lim _{r \rightarrow R_{m}^{*-}} \psi(r, z)=0$,

$$
\begin{aligned}
& \lim _{r \rightarrow R_{m}^{*-}} \frac{\psi^{*}(r, z)}{\psi(r, z)}=\lim _{r \rightarrow R_{m}^{*-}} \frac{\left(\partial \psi^{*} / \partial r\right)(r, z)}{(\partial \psi / \partial r)(r, z)} \\
& =\lim _{r \rightarrow R_{m}^{*-}} \frac{(1+\beta)^{2} V^{2} / r f_{0}+(1+\beta) V}{V^{2} / r f_{0}+V} \\
& =\frac{\left(1+\beta_{2}\right)^{2} V_{m}^{2}\left(r_{m}^{*} / R_{m}^{*}\right)^{2 \alpha}+f_{0} R_{m}^{*}\left(1+\beta_{2}\right) V_{m}\left(r_{m}^{*} / R_{m}^{*}\right)^{\alpha}}{V_{m}^{2}\left(r_{m}^{*} / R_{m}^{*}\right)^{2 \alpha}+f_{0} R_{m}^{*} V_{m}\left(r_{m}^{*} / R_{m}^{*}\right)^{\alpha}} \\
& =1+\frac{\left(\beta_{2}^{2}+2 \beta_{2}\right) V_{m}^{2}\left(r_{m}^{*} / R_{m}^{*}\right)^{2 \alpha}+f_{0} R_{m}^{*} \beta_{2} V_{m}\left(r_{m}^{*} / R_{m}^{*}\right)^{\alpha}}{V_{m}^{2}\left(r_{m}^{*} / R_{m}^{*}\right)^{2 \alpha}+f_{0} R_{m}^{*} V_{m}\left(r_{m}^{*} / R_{m}^{*}\right)^{\alpha}} .
\end{aligned}
$$

Therefore, $\beta_{2}=0$ is a necessary and sufficient condition to make

$$
\lim _{r \rightarrow R_{m}^{*-}} \frac{\psi^{*}(r, z)}{\psi(r, z)}=1 .
$$


From the continuity of wind, it can be deduced that

$$
\begin{aligned}
\beta_{2}(z) & =\sqrt{\frac{\left\langle u^{2}\left(R_{m}^{*}(z), \theta, z\right)+v^{2}\left(R_{m}^{*}(z), \theta, z\right)\right\rangle}{\left\langle u^{2}\left(R_{m}(z), \theta, z\right)+v^{2}\left(R_{m}(z), \theta, z\right)\right\rangle}}-1 \\
& =0 .
\end{aligned}
$$

$R_{m}^{*}(z)=R_{m}(z)$ can guarantee the above equations. The continuity of other variables can be deduced from the continuity of pressure and temperature. In conclusion, the vortex boundary conditions for all of the variables require that $R_{m}^{*}(z)=R_{m}(z)$.

\section{Evaluations of the Vortex Initialization Scheme}

3.1. A Real Case Example: Super Typhoon Phanfone (2014). The super typhoon Phanfone (2014) is used as an example to illustrate the effects of the vortex initialization scheme on the initial conditions. The initial time is 00:00:00 (UTC) on 1 Oct, 2014. In Figures 1-4, the surface wind speed $\mathrm{WSP}_{s}$, surface pressure $p_{s}$, surface temperature $T_{s}$, and surface water vapor mixing ratio $q_{s}$ before and after initialization are compared in the parent domain (D01), the middle domain (D02), and the inner domain (D03). The parent domain (D01) has a horizontal resolution of $27 \mathrm{~km}$, with $199 \times 172$ grids; the middle nest domain (D02) has a resolution of $9 \mathrm{~km}$, with $301 \times 223$ grids; and the inner domain (D03) has a resolution of $3 \mathrm{~km}$, with $469 \times 370$ grids. The domains are shown in Figure 5. The variables in the inner domain are identically interpolated back to the middle domain and the parent domain. The modified RMW $r_{m}^{*}\left(z_{\max }\right)$ is set as $90 \mathrm{~km}$ (the sensitivity of this parameter is discussed later); the observational max wind speed $V_{\text {obs }}\left(z_{\max }\right)$ is set as $60 \mathrm{kt}$. After initialization, strong wind around the typhoon center can be observed in Figure 1(f). Before initialization, the eye wall of the typhoon is not formed (Figure 1(e)). At the initial time, the observational CSLP is $975 \mathrm{hPa}$, whereas the CSLP before initialization is higher than $990 \mathrm{hPa}$ (Figure 2(e)), which needs to be intensified. As shown in Figures 3 and 4, the temperature and moisture fields at the initial time are both intensified simultaneously.

3.2. Typhoon Cases and Evaluation Metrics. Simulations were conducted for severe and super typhoon cases in the northwestern Pacific during 2013-2014. The simulated cases are severe typhoon Wutip (2013), severe typhoon Fitow (2013), severe typhoon Krosa (2013), super typhoon Genevieve (2014), super typhoon Phanfone (2014), and super typhoon Nuri (2014), because the initial MWSP derived from FNL is not strong enough for these cases. The cases are listed in chronological order, and the simulation periods are also listed in Table 1 . The simulations start half a day before the typhoon stage. The duration for most simulations is 96 hours, except for Genevieve (for which the observational data starts from the 180th meridian) and Wutip (which ends with dissipation). The simulation results are compared with the observational data from the Hong Kong Observatory (HKO).
TABLE 1: Simulation periods.

\begin{tabular}{lccc}
\hline Name & Start time (UTC) & End time (UTC) & Length (h) \\
\hline Wutip & 00:00, 28 Sep, 2013 & $12: 00,30$ Sep, 2013 & 60 \\
Fitow & 12:00, 2 Oct, 2013 & 12:00, 6 Oct, 2013 & 96 \\
Krosa & 00:00, 30 Oct, 2013 & 00:00, 3 Nov, 2013 & 96 \\
Genevieve & 12:00, 9 Aug, 2014 & 12:00, 11 Aug, 2014 & 48 \\
Phanfone & 00:00, 1 Oct, 2014 & 00:00, 5 Oct, 2014 & 96 \\
Nuri & 00:00, 1 Nov, 2014 & 00:00, 5 Nov, 2014 & 96 \\
\hline
\end{tabular}

The variables to be compared are track, CSLP, and maximum surface wind speed (MWSP). The observational data were available every 6 hours. Therefore, the simulated data are compared with observations every 6 hours. Consider the time series of observational data $O_{i}$ and simulated data $S_{i}, 1 \leq i \leq$ $N$. $N$ is the number of time points to be compared, that is, (length of simulation) $/ 6+1$. The definition of absolute error (AE) in this paper for $i$-th time point is $\left|S_{i}-O_{i}\right|$, which is slightly different from the traditional definition $\left(S_{i}-O_{i}\right)$. The mean absolute error (MAE) is defined as

$$
\text { MAE }=\frac{1}{N} \sum_{i=1}^{N}\left|S_{i}-O_{i}\right| .
$$

The MAE is affected equally by all of the time points during the simulation period and can be used as a variable to evaluate a simulation's overall performance. The improvements of the initialization scheme are evaluated by

$$
\begin{aligned}
& \text { improvement of } \mathrm{AE}=\frac{\mathrm{AE}^{\mathrm{no}}-\mathrm{AE}^{\text {init }}}{\mathrm{AE}^{\mathrm{no}}} \times 100 \%, \\
& \text { improvement of } \mathrm{MAE} \\
& \quad=\frac{\mathrm{MAE}^{\mathrm{no}}-\mathrm{MAE}^{\text {init }}}{\mathrm{MAE}^{\mathrm{no}}} \times 100 \%,
\end{aligned}
$$

where "no" signifies no initialization and "init" signifies initialization. In the figures and tables, the units for track, CSLP, and MWSP are $\mathrm{km}, \mathrm{hPa}$, and $\mathrm{m} \mathrm{s}^{-1}$, respectively.

3.3. WRF Configurations. Numerical simulations were conducted by WRF-ARW v3.5.1. As an example, the domains for super typhoon Phanfone (2014) are illustrated in Figure 5. For the other cases, the domains are horizontally shifted by changing the reference latitude and reference longitude in the WRF preprocessing system (WPS). For each case, D03 is designed to make the initial vortex located at its right-bottom corner, which helps the inner domain to cover the tracks as far as possible. All of the domains have 39 vertical layers. The Lambert projection method is applied. The time steps are $120 \mathrm{~s}, 40 \mathrm{~s}$, and 40/3 s for D01, D02, and D03, respectively. To maintain stability, the time step in seconds must be $6 \times \Delta x$ or less, where $\Delta x$ is the grid size in $\mathrm{km}$. The current settings for time steps can satisfy the stability constraint and control the computational cost at the same time. The pressure at the top of the domains is set as $50 \mathrm{hPa}$, which is enough to reach 


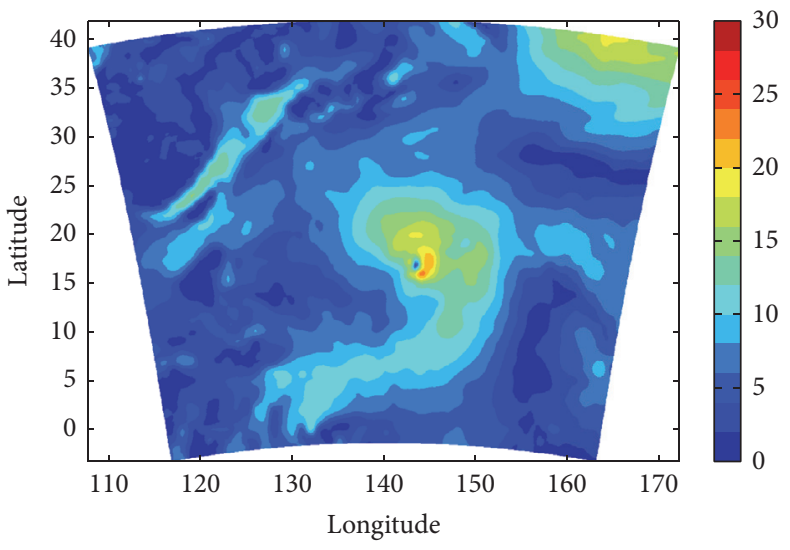

(a)

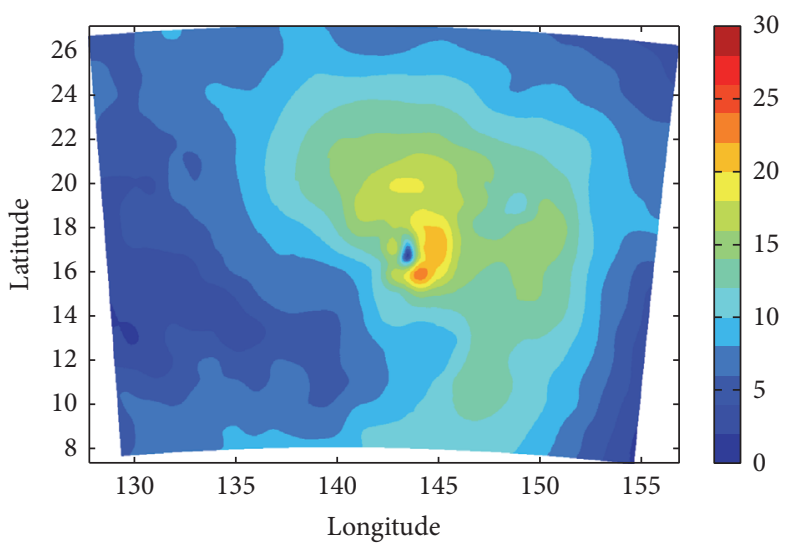

(c)

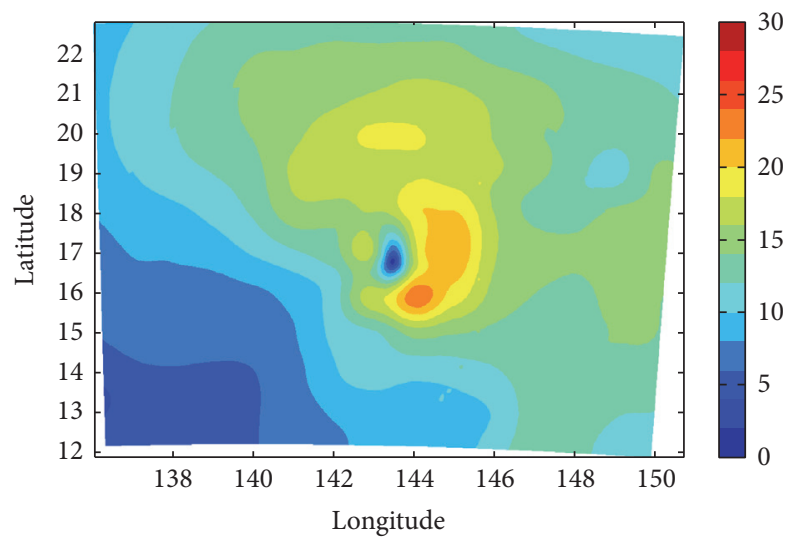

(e)

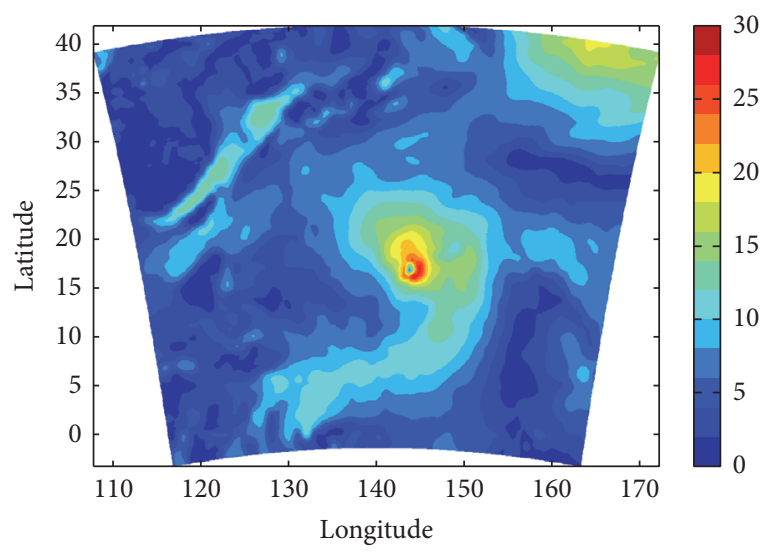

(b)

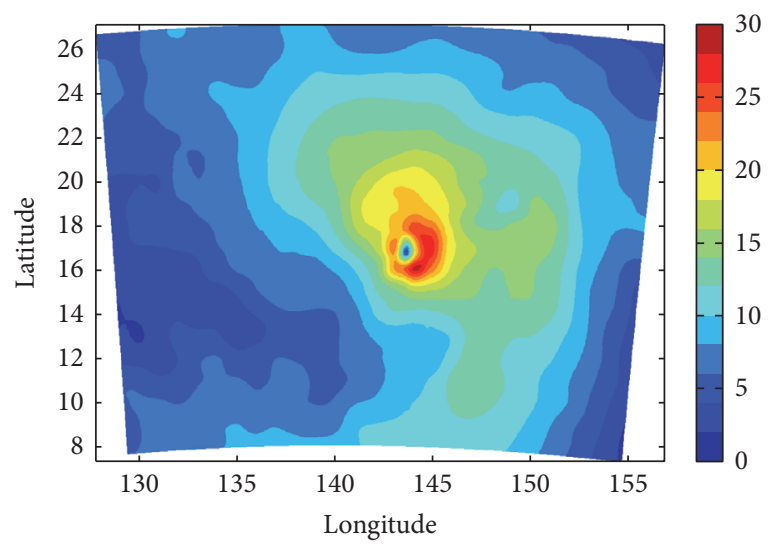

(d)

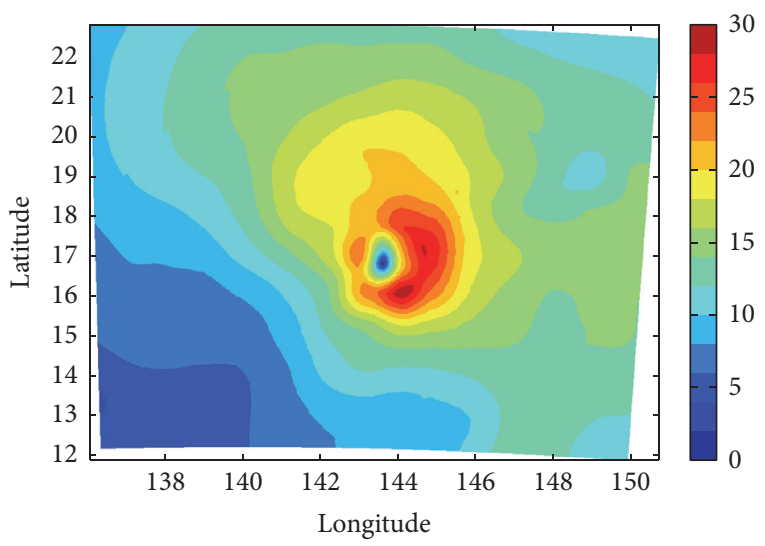

(f)

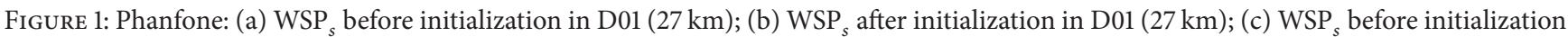

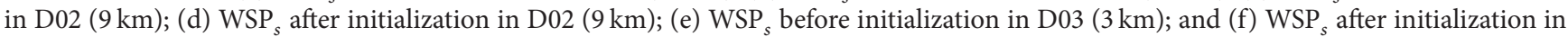
D03 $(3 \mathrm{~km})$. The unit in these figures is $\mathrm{m} / \mathrm{s}$.

the tropopause height for tropical cyclone simulations. Twoway nesting is used for all of the simulations. Time varying sea surface temperature, sea-ice, vegetation fraction, and albedo are used. No data assimilations are used. For physical parameterizations, the YSU planetary boundary layer scheme is used. The microphysics scheme is the WSM6 scheme [16]. The long-wave and short-wave radiation schemes are the
RRTMG schemes [17]. The Grell-Freitas cumulus scheme [18] is applied in D01 and D02.

The initial conditions and boundary conditions are generated by the FNL data (6-hourly, 1-degree resolution). The initial conditions can be further modified by the vortex initialization scheme, if the initial MWSP is not strong enough. The configurations of all the six cases simulated with 


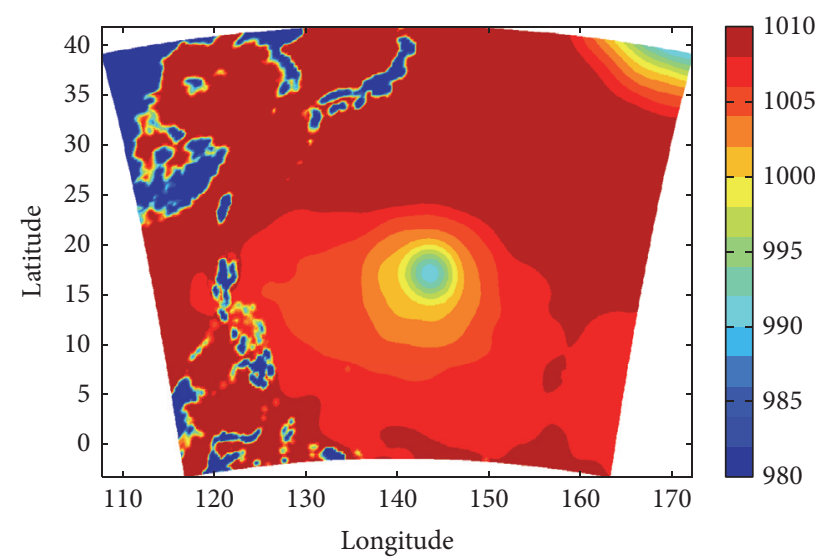

(a)

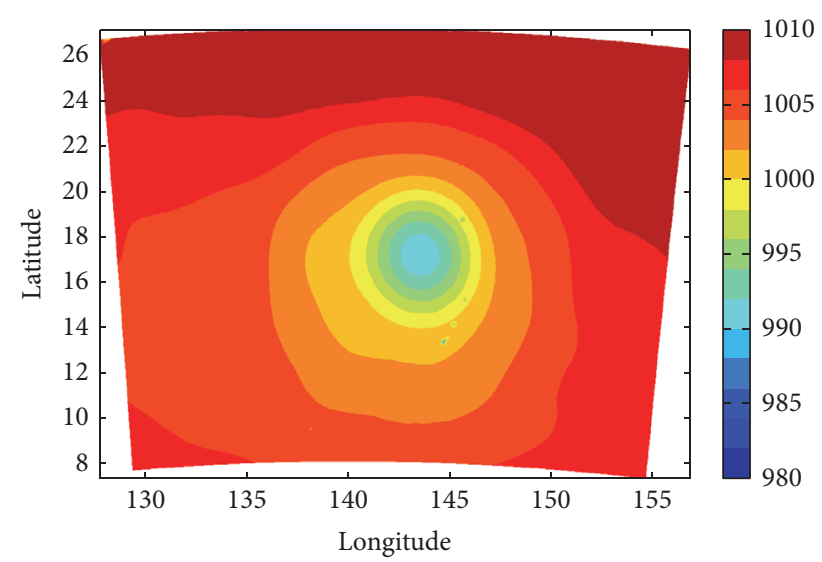

(c)

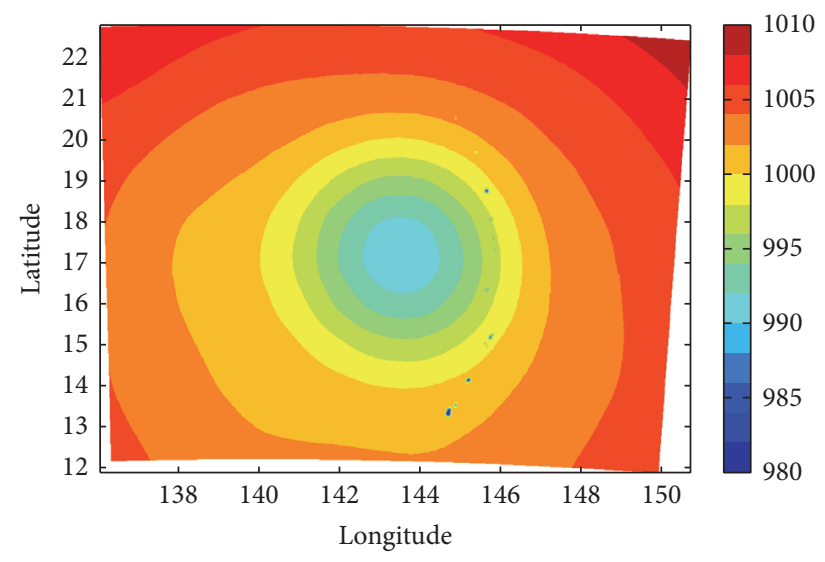

(e)

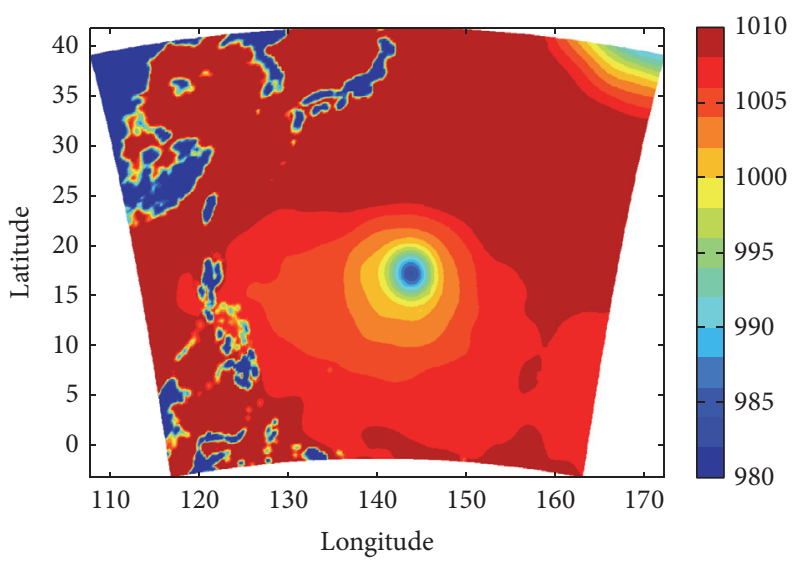

(b)

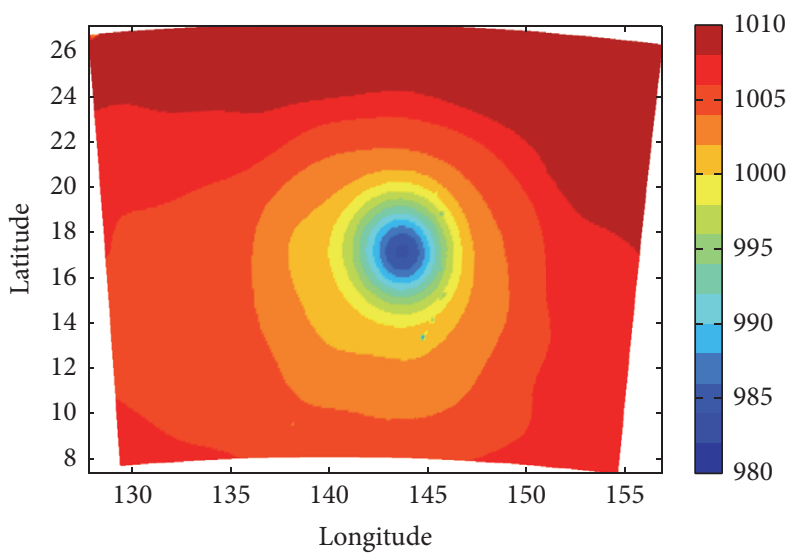

(d)

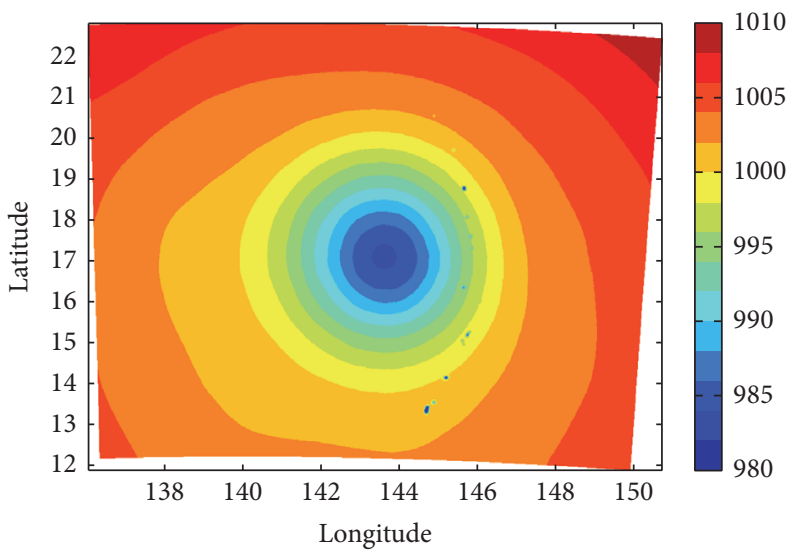

(f)

Figure 2: Phanfone: (a) $p_{s}$ before initialization in D01 (27 km); (b) $p_{s}$ after initialization in D01 (27 km); (c) $p_{s}$ before initialization in D02 $(9 \mathrm{~km})$; (d) $p_{s}$ after initialization in D02 $(9 \mathrm{~km})$; (e) $p_{s}$ before initialization in D03 $(3 \mathrm{~km})$; and (f) $p_{s}$ after initialization in D03 $(3 \mathrm{~km})$. The unit in these figures is $\mathrm{hPa}$.

the vortex initialization scheme are summarized in Table 2. Although the observational RMW is currently unavailable, the input parameter RMW in the initialization scheme has a low sensitivity. As shown in Figure 6, the super typhoon Phanfone (2014) was simulated with three RMW values (80, 90 , and $100 \mathrm{~km})$. From the time series of track, CSLP, and MWSP, it can be observed that the simulation results are close to each other for different RMW values.

\section{Results and Analysis}

For Wutip, Fitow, Krosa, Genevieve, Phanfone, and Nuri, comparisons between without and with initialization are made for track, CSLP, and MWSP. The main metric to evaluate the simulations is the MAE, which is defined in (57). If the FNL data for one case can provide sufficiently strong initial MWSP but cannot provide a low enough CSLP, the 


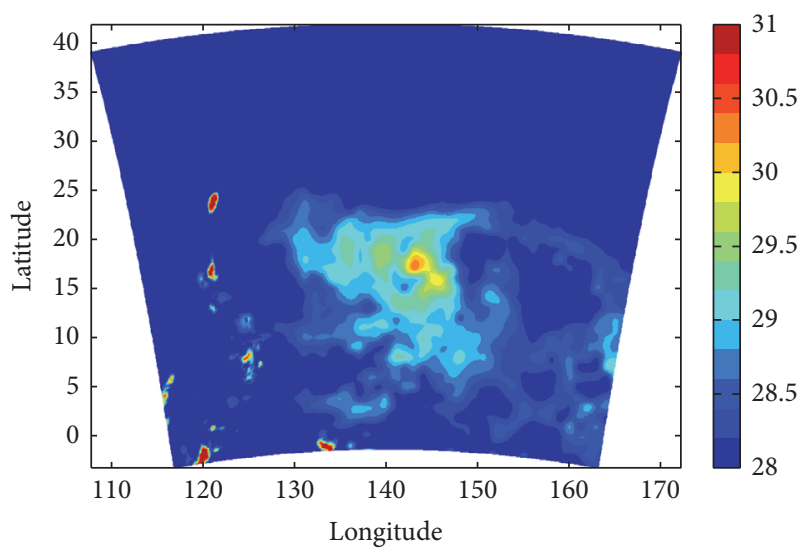

(a)

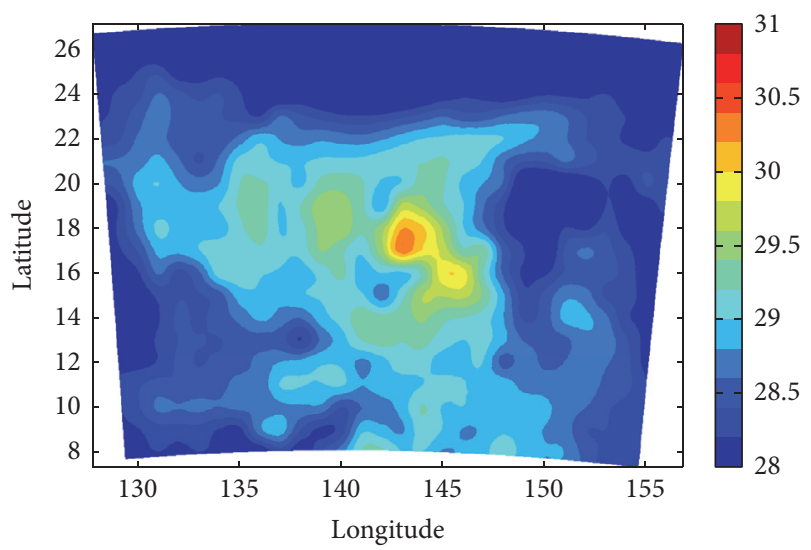

(c)

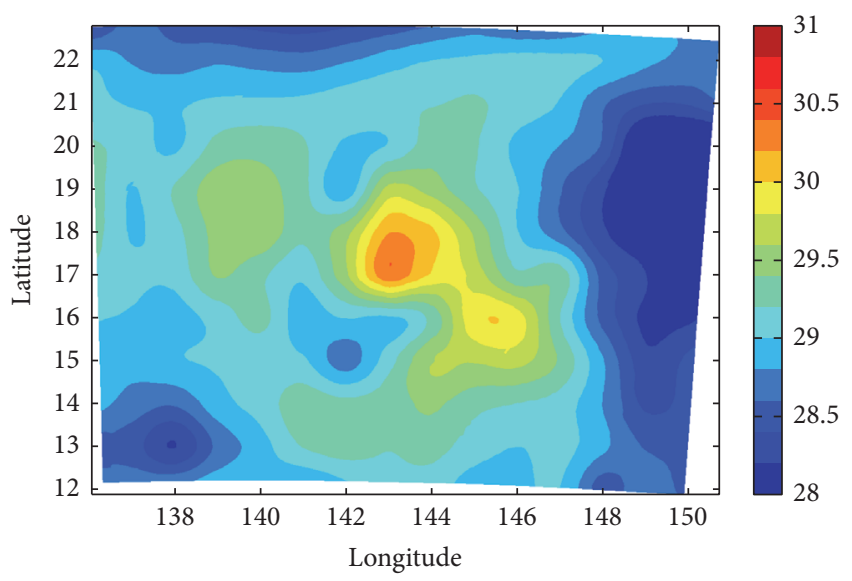

(e)

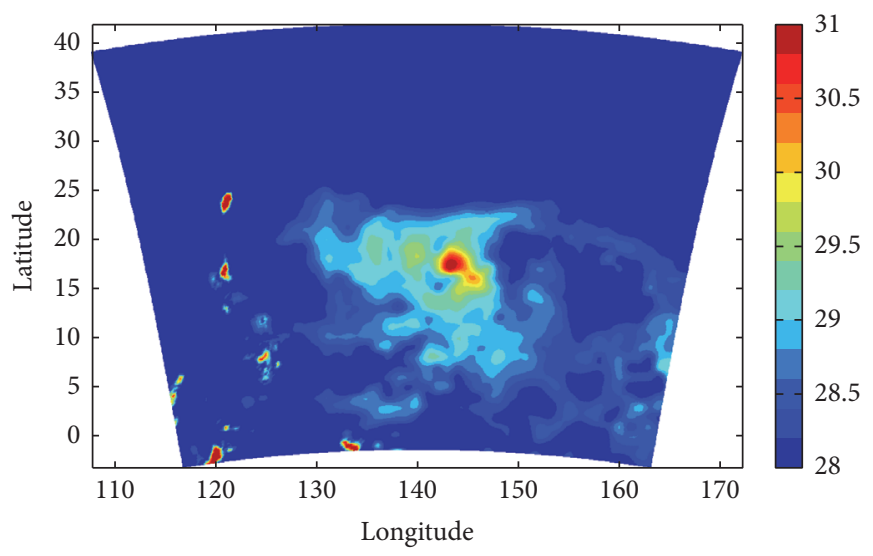

(b)

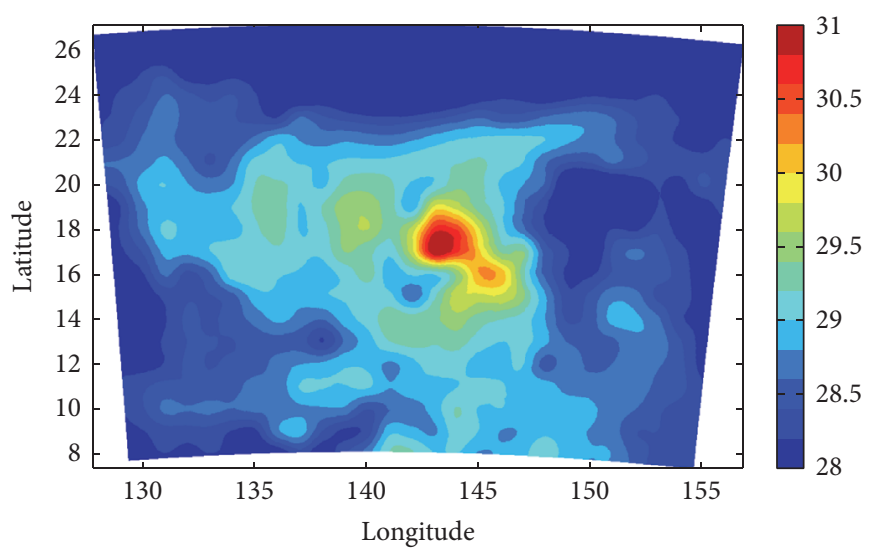

(d)

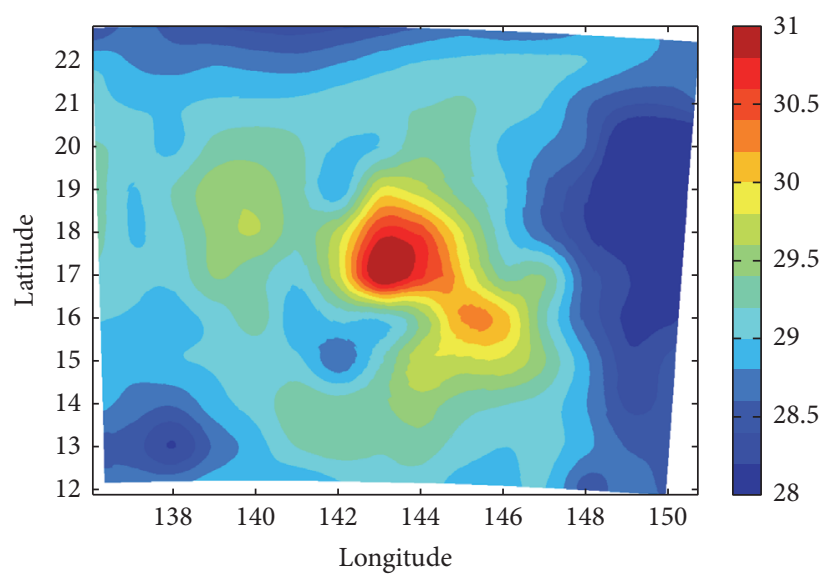

(f)

Figure 3: Phanfone: (a) $T_{s}$ before initialization in D01 (27 km); (b) $T_{s}$ after initialization in D01 (27 km); (c) $T_{s}$ before initialization in D02 $(9 \mathrm{~km})$; (d) $T_{s}$ after initialization in D02 $(9 \mathrm{~km}) ;(\mathrm{e}) T_{s}$ before initialization in D03 $(3 \mathrm{~km})$; and (f) $T_{s}$ after initialization in D03 (3 km). The unit in these figures is ${ }^{\circ} \mathrm{C}$.

vortex initialization scheme will still not be used because our scheme uses the gradient wind balance to deduce the pressure field from the wind field. If the wind field from FNL is stronger than the observation, the intensity correction factor will be negative, which further increases the center pressure. In the following subsections, we analyze the casespecific results and summarize the overall performance.
4.1. Analysis of Case-Specific Results. In Table 3, the improvements of track, CSLP, and MWSP made by the vortex initialization scheme are illustrated for each case. The vortex initialization scheme has little impact on the track prediction results. This is because the track is largely dependent on the large-scale analysis, while vortex initialization can only modify the vortex part. For Fitow and Genevieve, the 


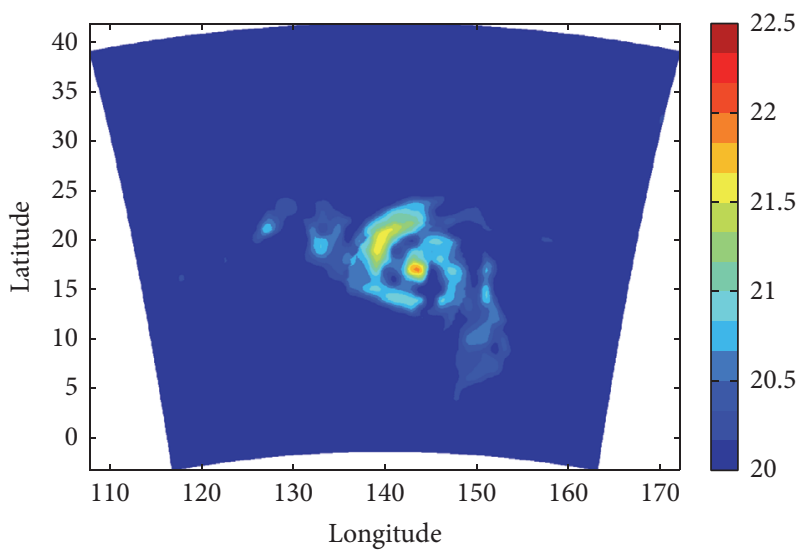

(a)

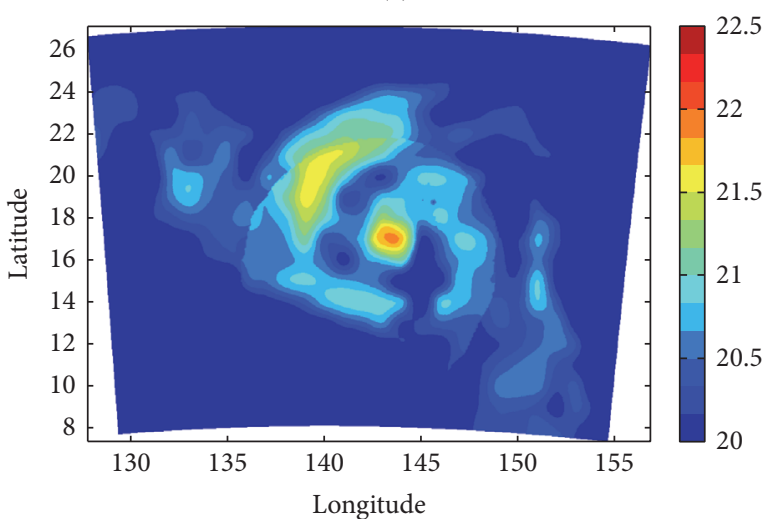

(c)

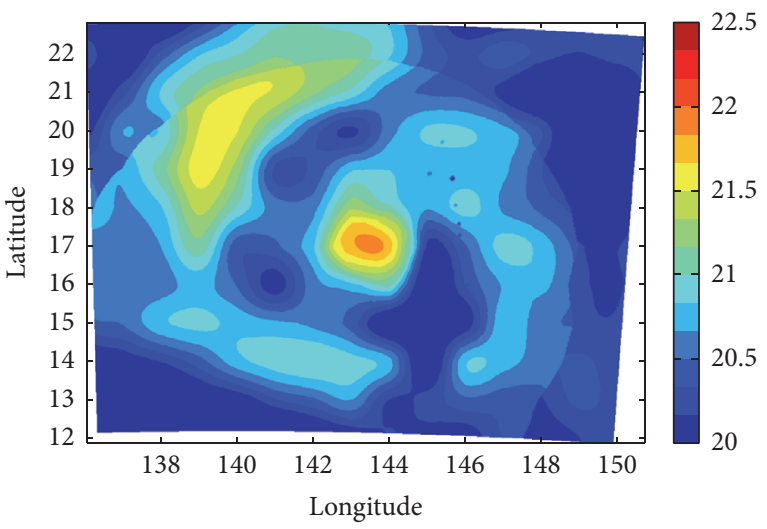

(e)

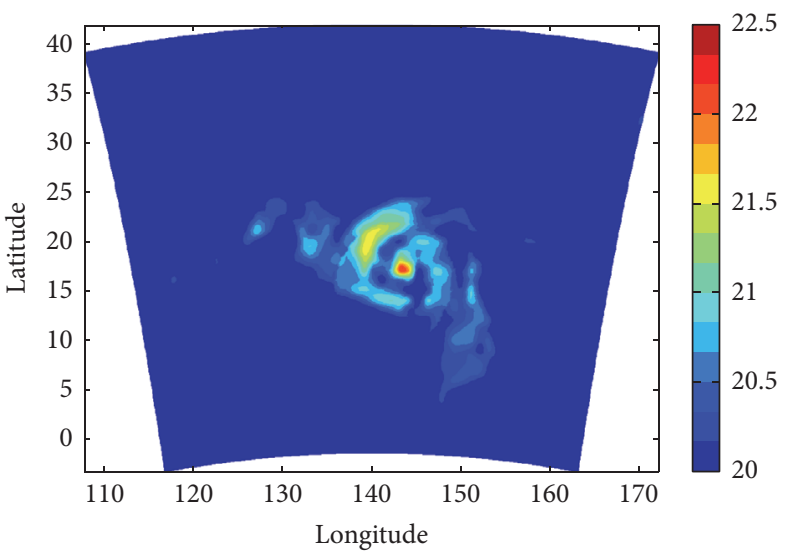

(b)

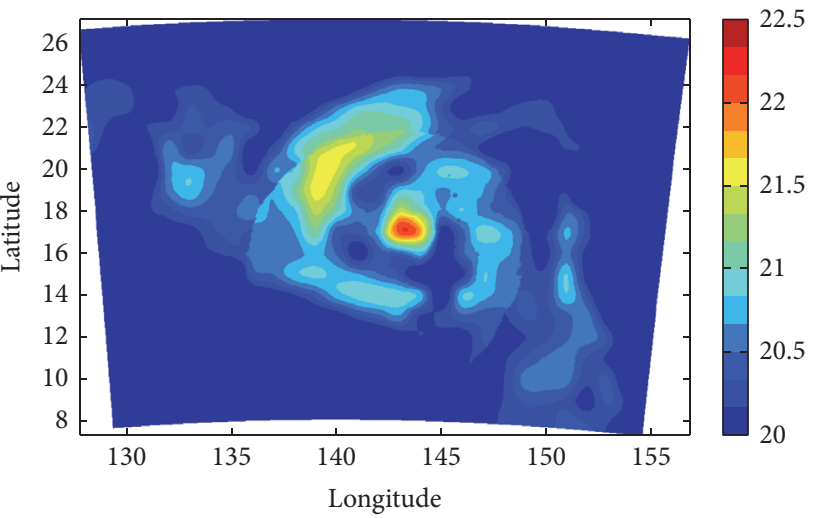

(d)

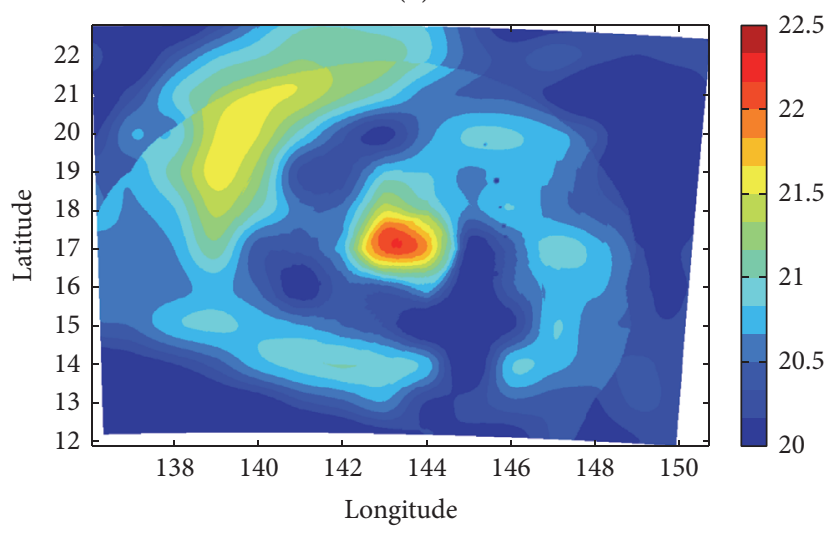

(f)

FIGURE 4: Phanfone: (a) $q_{s}$ before initialization in D01 (27 km); (b) $q_{s}$ after initialization in D01 (27 km); (c) $q_{s}$ before initialization in D02 $(9 \mathrm{~km}) ;(\mathrm{d}) q_{s}$ after initialization in D02 $(9 \mathrm{~km}) ;(\mathrm{e}) q_{s}$ before initialization in D03 $(3 \mathrm{~km})$; and (f) $q_{s}$ after initialization in D03 (3 km). The unit in these figures is $\mathrm{g} / \mathrm{kg}$.

scheme increases the MAE of track by $4.6 \%$ and $3.4 \%$, respectively. For Wutip and Nuri, the scheme results in larger improvements in the MAE of track. In general, the vortex initialization scheme improves the CSLP and MWSP prediction results. The scheme reduces the MAE of CSLP by $7.6 \%-30.2 \%$ and the MAE of MWSP by $7.9 \%-42.3 \%$.

4.2. Analysis of Overall Performance. In this section, the averaged terms are analyzed, with the averages taken for all the cases. Through the vortex initialization scheme, the averaged MAEs of track, CSLP, and MWSP are improved by $4.0 \%, 17.6 \%$, and $14.5 \%$, respectively (Table 4 ). In Table 4, averaged AEs and MAEs are reported. With the exception of $24 \mathrm{~h}$ track, $48 \mathrm{~h}$ track, and $72 \mathrm{~h}$ CSLP, the scheme improves all of the variables (Table 4). Table 5 presents comparisons between our scheme and some other schemes. In the column "our scheme," a value in bold is the minimum of its row (which means that our scheme provides the best performance of the corresponding metric among all the schemes). 
TABLE 2: Configurations in the vortex initialization scheme.

\begin{tabular}{lccc}
\hline Name & $\begin{array}{c}\text { Max } \\
\text { observational } \\
\text { wind speed } \\
(\mathrm{kt})\end{array}$ & $\begin{array}{c}\text { Radius of } \\
\text { max wind } \\
(\mathrm{km})\end{array}$ & Initial location \\
\hline Wutip & 60 & 90 & $16.4^{\circ} \mathrm{N}, 114.1^{\circ} \mathrm{E}$ \\
Fitow & 60 & 90 & $18.9^{\circ} \mathrm{N}, 129.9^{\circ} \mathrm{E}$ \\
Krosa & 60 & 90 & $16.2^{\circ} \mathrm{N}, 129.8^{\circ} \mathrm{E}$ \\
Genevieve & 100 & 75 & $22.7^{\circ} \mathrm{N}, 177.3^{\circ} \mathrm{E}$ \\
Phanfone & 60 & 90 & $16.9^{\circ} \mathrm{N}, 143.8^{\circ} \mathrm{E}$ \\
Nuri & 60 & 90 & $13.0^{\circ} \mathrm{N}, 133.9^{\circ} \mathrm{E}$ \\
\hline
\end{tabular}

TABLE 3: Improvements of track, CSLP, and MWSP by the vortex initialization scheme.

\begin{tabular}{lccc}
\hline Name & $\begin{array}{c}\text { Improvements } \\
\text { of track }\end{array}$ & $\begin{array}{c}\text { Improvements } \\
\text { of CSLP }\end{array}$ & $\begin{array}{c}\text { Improvements } \\
\text { of MWSP }\end{array}$ \\
\hline Wutip & $13.8 \%$ & $30.2 \%$ & $42.3 \%$ \\
Fitow & $-4.6 \%$ & $7.6 \%$ & $21.9 \%$ \\
Krosa & $6.8 \%$ & $21.0 \%$ & $13.2 \%$ \\
Genevieve & $-3.4 \%$ & $18.7 \%$ & $8.6 \%$ \\
Phanfone & $5.9 \%$ & $14.0 \%$ & $7.9 \%$ \\
Nuri & $10.7 \%$ & $18.7 \%$ & $10.3 \%$ \\
\hline
\end{tabular}

In Xiao et al. [10], the averaged track error of the bogus data assimilation (BDA) scheme is around $110 \mathrm{~km}$, whereas the averaged MAE of track simulated by our scheme is $114.2 \mathrm{~km}$; the averaged CSLP error is around $17 \mathrm{hPa}$ (our scheme: $11.6 \mathrm{hPa}$, as shown in Table 5); and the averaged MWSP is around $9 \mathrm{~m} / \mathrm{s}$ (our scheme: $5.7 \mathrm{~m} / \mathrm{s}$ ). The scheme in Kwon and Cheong [7] decreases the $48 \mathrm{~h}$ track error to $173.9 \mathrm{~km}$ on average (our scheme: $103.4 \mathrm{~km}$ ); the $48 \mathrm{~h}$ error for CSLP is improved from $21.2 \mathrm{hPa}$ to $10.0 \mathrm{hPa}$ on average (our scheme: $15.0 \mathrm{hPa}$ ); and the $24 \mathrm{~h}$ CSLP error is improved from $37.3 \mathrm{hPa}$ to $11.2 \mathrm{hPa}$ on average (our scheme: $9.2 \mathrm{hPa}$ ). In Hsiao et al. [11], the track errors are 208, 95, and $312 \mathrm{~km}$ for the with-relocation runs at 24,48 , and $72 \mathrm{~h}$, respectively (our scheme: 69.7, 103.4, and $163.4 \mathrm{~km}$ at 24, 48, and $72 \mathrm{~h}$ ). In Cha and Wang [12], for the MAEs averaged for all forecasts, the dynamic initialization scheme reduces the absolute track error to $103.3 \mathrm{~km}$ (our scheme: $114.2 \mathrm{~km}$ ) and reduces the absolute MWSP error to $7.3 \mathrm{~m} / \mathrm{s}$ (our scheme: $5.7 \mathrm{~m} / \mathrm{s}$ ).

\section{Summary and Conclusions}

In this paper, a new vortex initialization scheme is developed and tested. Through this scheme, a new framework of initial vortex construction is introduced, which starts from 3D wind construction. The other variables, such as pressure, temperature, and humidity, are also constructed on the basis of dynamical and thermodynamical equations.

The scheme requires only observational data for the center location and max wind speed in addition to global analysis data. One advantage of this scheme is that it can estimate a $3 \mathrm{D}$
TABLE 4: Averaged terms and improvements of track, CSLP, and MWSP by the vortex initialization scheme.

\begin{tabular}{lccc}
\hline $\begin{array}{l}\text { Averaged AE } \\
\text { terms }\end{array}$ & $\begin{array}{c}\text { Without } \\
\text { initialization }\end{array}$ & $\begin{array}{c}\text { With } \\
\text { initialization }\end{array}$ & Improvement \\
\hline $24 \mathrm{~h}$ track & 58.2 & 69.7 & $-19.8 \%$ \\
$48 \mathrm{~h}$ track & 99.3 & 103.4 & $-4.2 \%$ \\
$72 \mathrm{~h}$ track & 178.3 & 163.4 & $8.4 \%$ \\
96 h track & 365.6 & 326.3 & $10.8 \%$ \\
MAE of track & 119.0 & 114.2 & $4.0 \%$ \\
\hline 24h CSLP & 13.9 & 9.2 & $33.6 \%$ \\
48 h CSLP & 15.8 & 15.0 & $4.8 \%$ \\
72 h CSLP & 12.8 & 12.9 & $-0.7 \%$ \\
96 h CSLP & 14.0 & 14.0 & $0.1 \%$ \\
MAE of & 14.1 & 11.6 & $17.6 \%$ \\
CSLP & 6.6 & 6.5 & $2.1 \%$ \\
\hline 24h MWSP & 8.1 & 7.0 & $13.5 \%$ \\
48 h MWSP & 7.3 & 6.8 & $7.0 \%$ \\
72 h MWSP & 5.0 & 3.2 & $35.2 \%$ \\
96h MWSP & 6.7 & 5.7 & $14.5 \%$ \\
MAE of & & & \\
MWSP & & &
\end{tabular}

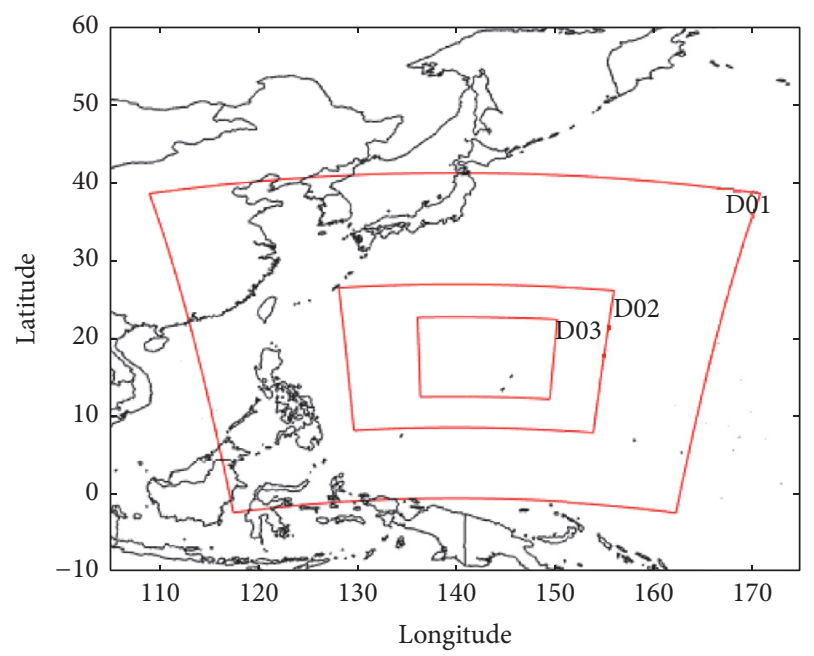

FIGURE 5: WRF domains for the super typhoon Phanfone (2014) D01 $(27 \mathrm{~km}) ; \mathrm{D} 02(9 \mathrm{~km})$; and D03 $(3 \mathrm{~km})$.

TC vortex with only little observational data. The derivation of the scheme is mainly based on the dynamical balance constraints. Therefore, the scheme is highly analytic, which makes it satisfy the vortex boundary conditions and with low computational cost. Some highlights of this work include (1) determination of the intensity correction factor at the vortex boundary $\beta_{2}$ (23); (2) using the absolute angular momentum at the RMW to derive the RMW function $r_{m}^{*}(z)$ (28); (3) explicit analytic formulations of the gradient wind stream functions ((36a), (36b), (36c), (37a), (37b), and (37c)), which are useful for improving the computational speed of pressure construction; (4) the construction of temperature and 
TABLE 5: Comparisons of our scheme with other schemes.

\begin{tabular}{|c|c|c|c|c|c|}
\hline Metrics $\backslash$ schemes & Xiao et al. [10] & Kwon and Cheong [7] & Hsiao et al. [11] & Cha and Wang [12] & Our scheme \\
\hline $24 \mathrm{~h} \mathrm{track}$ & - & - & 208 & - & 69.7 \\
\hline $48 \mathrm{~h}$ track & - & 173.9 & 95 & - & 103.4 \\
\hline $72 \mathrm{~h}$ track & - & - & 312 & - & 163.4 \\
\hline MAE of track & 110 & - & - & 103.3 & 114.2 \\
\hline 24 h CSLP & - & 11.2 & - & - & 9.2 \\
\hline $48 \mathrm{~h} \mathrm{CSLP}$ & - & 10.0 & - & - & 15.0 \\
\hline MAE of CSLP & 17 & - & - & - & 11.6 \\
\hline MAE of MWSP & 9 & - & - & 7.3 & 5.7 \\
\hline
\end{tabular}

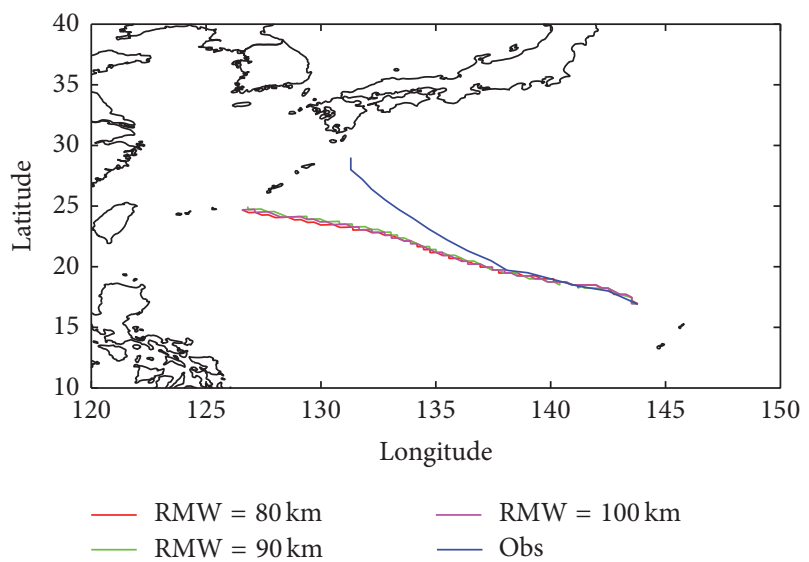

(a)

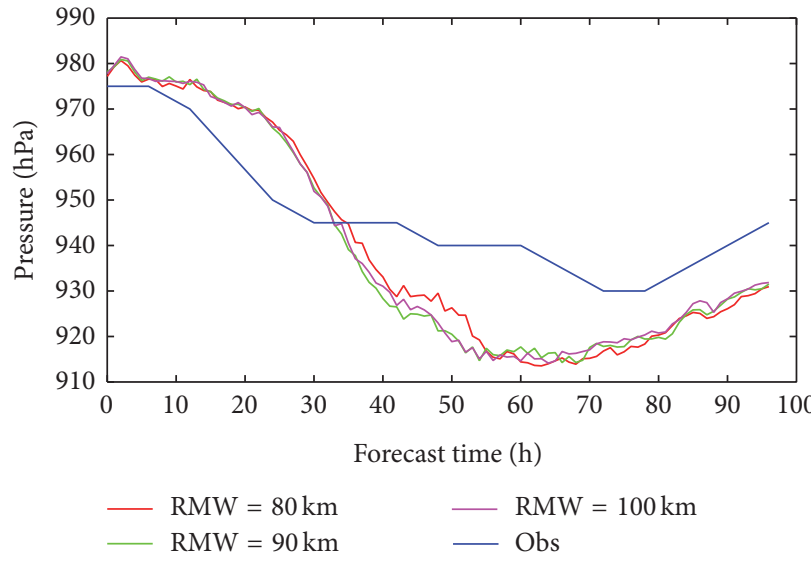

(b)

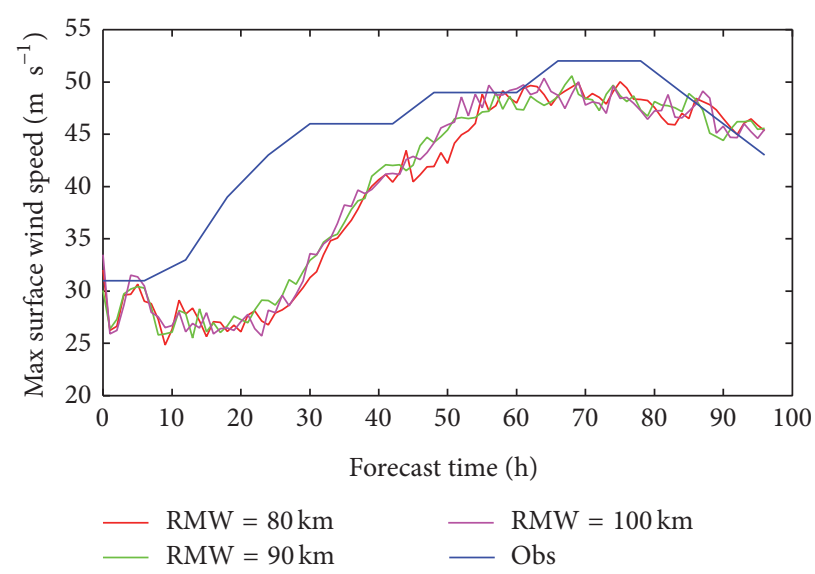

(c)

FIGURE 6: Phanfone: simulation results with RMW = 80, 90 and $100 \mathrm{~km}$ (a) track; (b) CSLP; (c) MWSP.

humidity is unified in (50); and (5) discussion of the vortex boundary continuity (a necessary and sufficient condition of vortex boundary continuity under the framework of this scheme is given in Section 2.5).

Some future work includes improving the parameters, for example, $\gamma$ and $L$ introduced in (6), which is the estimation for vertical profile of tangential wind speed. One potential weakness of the scheme is about the RMW, which cannot be obtained from observations currently.

The scheme was evaluated via simulations for severe and super typhoons in the northwestern Pacific. The scheme can provide a better prediction of typhoon intensity: the averaged MAEs of CSLP and MWSP are improved by $17.6 \%$ and $14.5 \%$, respectively. Compared with other methods and initialization 
schemes, the scheme can provide the best performance for the averaged MAEs of CSLP and MWSP.

\section{Acronyms}

$\begin{array}{ll}\text { AE: } & \text { Absolute error } \\ \text { CSLP: } & \text { Center sea-level pressure } \\ \text { FNL: } & \text { NCEP final analyses } \\ \text { GFS: } & \text { Global forecast system } \\ \text { HKO: } & \text { Hong Kong Observatory } \\ \text { MAE: } & \text { Mean absolute error } \\ \text { MR: } & \text { Modified Rankine } \\ \text { MWSP: } & \text { Maximum surface wind speed } \\ \text { NCEP: } & \text { National Centers for Environmental } \\ & \text { Prediction } \\ \text { PBL: } & \text { Planetary boundary layer } \\ \text { RMW: } & \text { Radius of max wind } \\ \text { TC: } & \text { Tropical cyclone } \\ \text { UTC: } & \text { Coordinated Universal Time } \\ \text { WRF/ARW: } & \text { Weather research and } \\ & \text { forecasting/advanced research WRF } \\ \text { WPS: } & \text { WRF preprocessing system } \\ \text { WSP: } & \text { Surface wind speed. }\end{array}$

\section{Competing Interests}

The authors declare that there are no competing interests regarding the publication of this paper.

\section{Acknowledgments}

The authors appreciate the assistance of the HKO, which provided the meteorological data. This work was supported by NSFC/RGC, Grant N_HKUST631/05; NSFC-FD, Grant U1033001; and the RGC, Grant 16300715.

\section{References}

[1] R. L. Elsberry, T. D. B. Lambert, and M. A. Boothe, "Accuracy of Atlantic and Eastern North Pacific tropical cyclone intensity forecast guidance," Weather and Forecasting, vol. 22, no. 4, pp. 747-762, 2007.

[2] M. DeMaria, C. R. Sampson, J. A. Knaff, and K. D. Musgrave, "Is tropical cyclone intensity guidance improving?" Bulletin of the American Meteorological Society, vol. 95, no. 3, pp. 387-398, 2014.

[3] F. D. Marks, L. K. Shay, G. Barnes et al., "Landfalling tropical cyclones: forecast problems and associated research opportunities," Bulletin of the American Meteorological Society, vol. 79, no. 2, pp. 305-323, 1998.

[4] R. A. Anthes, Tropical Cyclones: Their Evolution, Structure and Effects, Meteorological Monographs, American Meteorological Society, 1982.

[5] X. Zou and Q. Xiao, "Studies on the initialization and simulation of a mature hurricane using a variational bogus data assimilation scheme," Journal of the Atmospheric Sciences, vol. 57, no. 6 , pp. 836-860, 2000.

[6] E. D. Rappin, D. S. Nolan, and S. J. Majumdar, "A highly configurable vortex initialization method for tropical cyclones," Monthly Weather Review, vol. 141, no. 10, pp. 3556-3575, 2013.
[7] I. H. Kwon and H. B. Cheong, "Tropical cyclone initialization with a spherical high-order filter and an idealized threedimensional bogus vortex," Monthly Weather Review, vol. 138, no. 4, pp. 1344-1367, 2010.

[8] K. A. Reed and C. Jablonowski, "An analytic vortex initialization technique for idealized tropical cyclone studies in AGCMs," Monthly Weather Review, vol. 139, no. 2, pp. 689-710, 2011.

[9] D. P. Stern and D. S. Nolan, "On the vertical decay rate of the maximum tangential winds in tropical cyclones," Journal of the Atmospheric Sciences, vol. 68, no. 9, pp. 2073-2094, 2011.

[10] Q. Xiao, L. Chen, and X. Zhang, "Evaluations of BDA scheme using the advanced research WRF (ARW) model," Journal of Applied Meteorology and Climatology, vol. 48, no. 3, pp. 680689, 2009.

[11] L.-F. Hsiao, C.-S. Liou, T.-C. Yeh et al., "A vortex relocation scheme for tropical cyclone initialization in advanced research WRF," Monthly Weather Review, vol. 138, no. 8, pp. 3298-3315, 2010.

[12] D.-H. Cha and Y. Wang, "A dynamical initialization scheme for real-time forecasts of tropical cyclones using the WRF model," Monthly Weather Review, vol. 141, no. 3, pp. 964-986, 2013.

[13] S. G. Gopalakrishnan, Q. Liu, T. Marchok et al., Hurricane Weather Research and Forecasting (HWRF) Model Scientic Documentation, Edited by L. Bernardet, Monthly Weather Review, 2010.

[14] S. J. Lord, "Abogusing system for vortex circulations in the National Meteorological Center global forecast model," in Proceedings of the 19th Conference on Hurricanes and Tropical Meteorology, pp. 328-330, American Meteorological Society, 1991.

[15] D. P. Stern and D. S. Nolan, "Reexamining the vertical structure of tangential winds in tropical cyclones: observations and theory," Journal of the Atmospheric Sciences, vol. 66, no. 12, pp. 3579-3600, 2009.

[16] S. Y. Hong and J. O. J. Lim, "The WRF single-moment 6class microphysics scheme (WSM6)," Journal of the Korean Meteorological Society, vol. 42, no. 2, pp. 129-151, 2006.

[17] M. J. Iacono, J. S. Delamere, E. J. Mlawer, M. W. Shephard, S. A. Clough, and W. D. Collins, "Radiative forcing by long-lived greenhouse gases: calculations with the AER radiative transfer models," Journal of Geophysical Research Atmospheres, vol. 113, no. 13, Article ID D13103, 2008.

[18] G. A. Grell and S. R. Freitas, "A scale and aerosol aware stochastic convective parameterization for weather and air quality modeling," Atmospheric Chemistry and Physics Discussions, vol. 13, no. 9, pp. 5233-5250, 2013. 

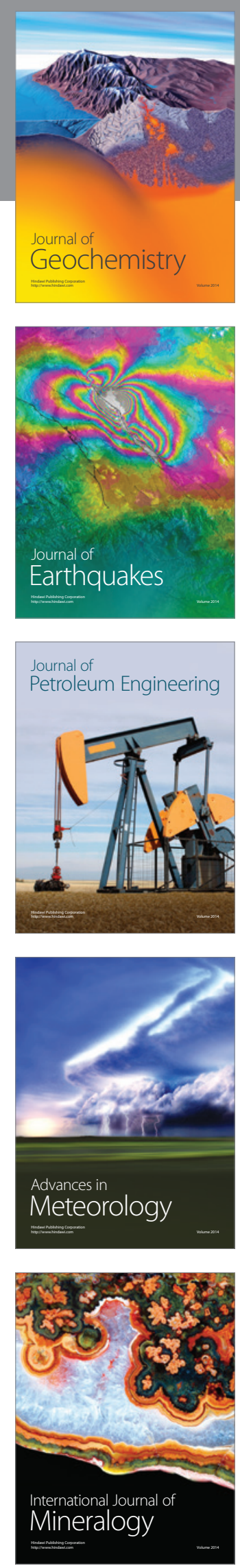
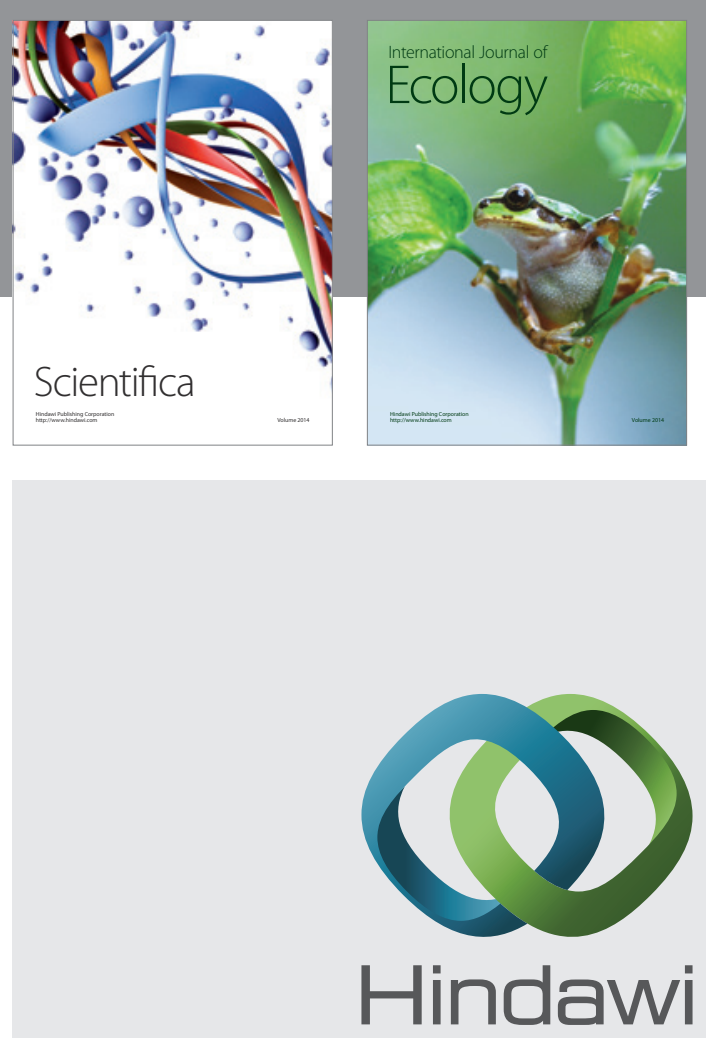

Submit your manuscripts at

https://www.hindawi.com
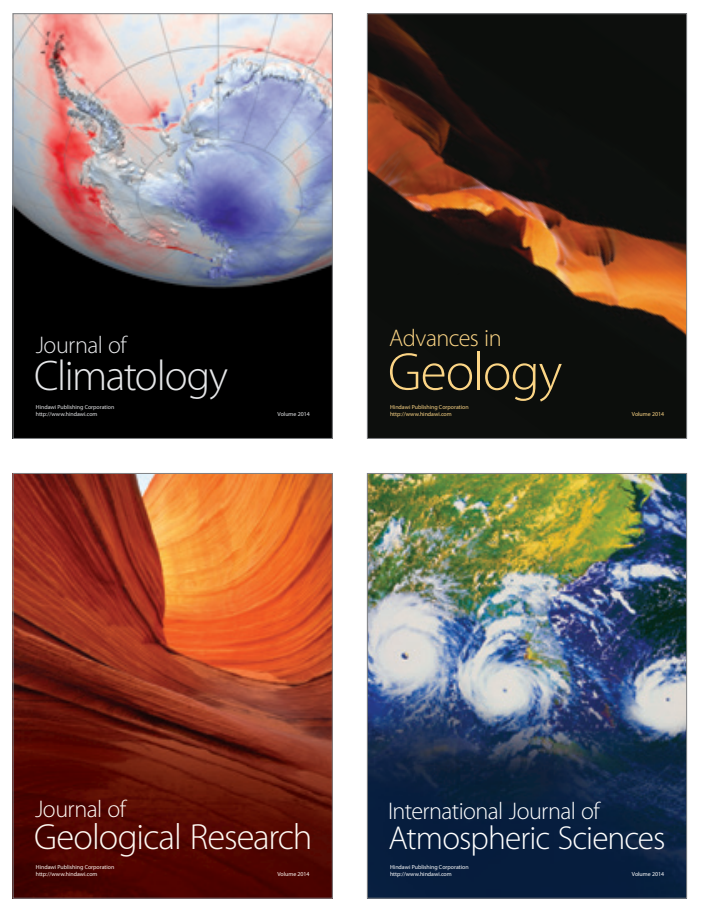

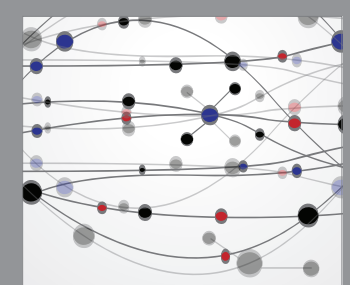

The Scientific

\section{World Journal}
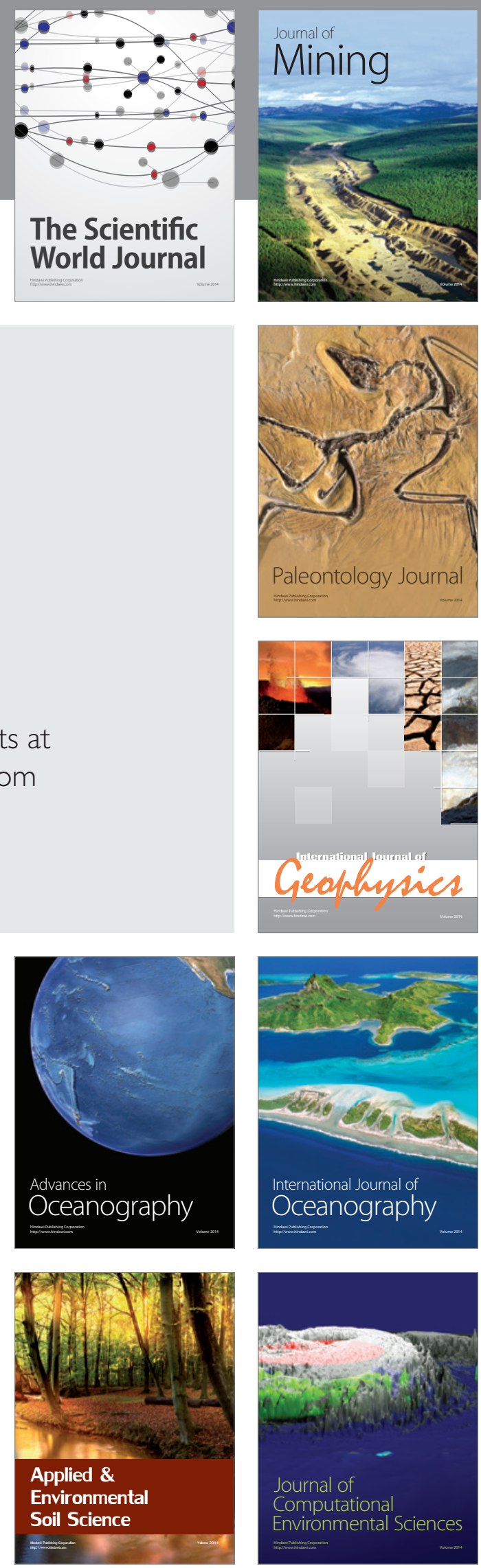